A linear optimization based method for data privacy in statistical tabular data

Jordi Castro José A. González

Dept. of Stat. and Operations Research

Univ. Politècnica de Catalunya

Barcelona, Catalonia

jordi.castro@upc.edu jose.a.gonzalez@upc.edu

Research Report UPC-DEIO DR 2017-02

September 2016; updated February 2017, May 2017

Report available from http://www-eio.upc.es/ j jcastro 



\title{
A linear optimization based method for data privacy in statistical tabular data
}

\author{
Jordi Castro* José A. González \\ Dept. of Statistics and Operations Research \\ Universitat Politècnica de Catalunya \\ Jordi Girona 1-3, 08034 Barcelona \\ jordi.castro@upc.edu jose.a.gonzalez@upc.edu
}

\begin{abstract}
National Statistical Agencies routinely disseminate large amounts of data. Prior to dissemination these data have to be protected to avoid releasing confidential information. Controlled tabular adjustment (CTA) is one of the available methods for this purpose. CTA formulates an optimization problem that looks for the safe table which is closest to the original one. The standard CTA approach results in a mixed integer linear optimization (MILO) problem, which is very challenging for current technology. In this work we present a much less costly variant of CTA that formulates a multiobjective linear optimization (LO) problem, where binary variables are pre-fixed, and the resulting continuous problem is solved by lexicographic optimization. Extensive computational results are reported using both commercial (CPLEX and XPRESS) and open source (Clp) solvers, with either simplex or interior-point methods, on a set of real instances. Most instances were successfully solved with the LO-CTA variant in less than one hour, while many of them are computationally very expensive with the MILO-CTA formulation. The interior-point method outperformed simplex in this particular application.
\end{abstract}

Keywords: linear optimization; interior-point methods; benchmarking; lexicographic optimization; data science; data privacy; statistical disclosure control;

AMS Subject Classification: 90C05, 90C29, 90C51, 90C90

\section{Introduction}

National Statistical Agencies (NSAs) release two different types of data: raw data, usually named microdata, and tabular data obtained by crossing two or more categorical variables of the microdata. NSAs face the tradeoff between publishing as much as possible information and at the same time to avoid that confidential information can be derived from data released. The field of statistical disclosure control comprises the set of available methods to reduce the disclosure risk. More details about this field can be found in the recent survey [6] and the monographs [20, 21].

Although tabular data report aggregated information - so individuals could be considered anonymized - there is a risk of disclosing confidential information. Tables of Figures 1 and 2 illustrate this situation with a simple case from [6]. Figure 1 shows a frequency table (i.e., a table providing the number of individuals within each cell) obtained by crossing variables "profession" and "municipality" of some microdata file. Figure 2 shows a magnitude table, i.e., one providing information about a third variable, overall salary (in $1000 €$ ) in this particular case. If cell $\left(M_{2}, P_{3}\right)$ of Figure 1 was 1 , then any person (including those who do not appear in the microdata file) would know the salary of this

* Corresponding author 


\begin{tabular}{c|c|c|c|c|c|c|}
\multicolumn{1}{c}{} & \multicolumn{1}{c}{$P_{1}$} & $P_{2}$ & $P_{3}$ & \multicolumn{1}{c}{$P_{4}$} & \multicolumn{1}{c|}{$P_{5}$} & \multicolumn{1}{c}{ TOTAL } \\
\cline { 2 - 7 }$M_{1}$ & 20 & 15 & 30 & 20 & 10 & 95 \\
\cline { 2 - 7 }$M_{2}$ & 72 & 20 & 1 or 2 & 30 & 10 & 133 \\
\cline { 2 - 7 }$M_{3}$ & 38 & 38 & 15 & 40 & 11 & 142 \\
\cline { 2 - 7 } TOTAL & 130 & 73 & 46 & 90 & 31 & 370 \\
\cline { 2 - 7 } & & & &
\end{tabular}

Figure 1.: Two-dimensional frequency table showing number of persons for each profession and municipality.

\begin{tabular}{c|c|c|c|c|c|c|}
\multicolumn{1}{c}{} & \multicolumn{1}{c}{$P_{1}$} & $P_{2}$ & $P_{3}$ & $P_{4}$ & $P_{5}$ & \multicolumn{1}{c}{ TOTAL } \\
\cline { 2 - 7 }$M_{1}$ & 360 & 450 & 720 & 400 & 360 & 2290 \\
\cline { 2 - 7 }$M_{2}$ & 1440 & 540 & 22 & 570 & 320 & 2892 \\
\cline { 2 - 7 }$M_{3}$ & 722 & 1178 & 375 & 800 & 363 & 3438 \\
\cline { 2 - 7 } TOTAL & 2522 & 2168 & 1117 & 1770 & 1043 & 8620 \\
\cline { 2 - 7 } & & & & &
\end{tabular}

Figure 2.: Two-dimensional magnitude table showing overall salary (in 1000€) for each profession and municipality.

individual is $22000 €$. This is named an external attacker scenario. If the value of this cell was 2 , any of these two individuals could compute the salary of the other, becoming internal attackers. Even if the value of this cell was larger, e.g. 4, if one of them had a salary of, e.g., $18000 €$, there would be a disclosure risk, since the contribution of the largest respondent could exceed some predefined percentage of the cell total; this cell would be reported as sensitive by the so-called dominance rule. Indeed, the set of sensitive cells is a priori obtained by applying some sensitivity rules. Actually, the two more used rules are the $(n, k)$ dominance rule ( $n$ individuals of a cell cannot contribute to more than a $k \%$ to the cell value) and the $p \%$ rule (the cell is considered sensitive if some individual can compute an estimate of the value of another individual within a $p \%$ precision). A detailed explanation of these rules is out of the scope of this work; additional details about them can be found in [20, 21].

If a table contains sensitive cells, NSAs have to apply some tabular data protection method prior to publication. In short, those methods, basically either suppress or perturb the table cell values. Formally, a tabular data protection method can be seen as a map $F$ such that $F(T)=T^{\prime}$, i.e., table $T$ is transformed to another table $T^{\prime}$. The two main requirements for $F$ are: (1) $T^{\prime}$ should be "safe", and (2) the quality of $T^{\prime}$ should be high (or equivalently, the information loss should be small), i.e., analysis made with $T^{\prime}$ and $T$ should be similar. The disclosure risk can be analyzed through the inverse map $T=F^{-1}\left(T^{\prime}\right)$ : if not available or difficult to compute by any data attacker, then we may guarantee that $F$ is safe [7].

As far as we know, the first publication describing a protection method for tabular data was [1]. Today, the most used method is likely cell suppression [5, 26], a non-perturbative method, where some cell values are removed from the table. Among the perturbative approaches we find controlled tabular adjustment (CTA) [4, 12, which is the focus of this work.

The goal of CTA - which will be formulated in Section 2 -is, given a table with any structure, to find the closest safe table to the original one, according to some distance. This is achieved by adding to the original table a vector of deviations (or perturbations) of minimum norm that makes the released table safe. Safety is guaranteed by imposing that sensitive cells in the perturbed table are far enough from the original value. This means the cell value is either above or below some certain values, which requires a binary variable for the disjunctive constraint of each sensitive cell. The minimum amount of 
above or below perturbations imposed to each sensitive cell are named, respectively, upper protection and lower protection levels. Changes in sensitive cells induce changes in the remaining ones to satisfy the value of marginal or total cells. This standard CTA method results in a difficult mixed integer linear optimization (MILO) problem.

CTA is one of the protection methods included and discussed in the recent monographs [20, 21]. Among the recent literature on CTA variants we find [9, 18]. Although CTA is not as widely used as cell suppression, it has been applied as one of the steps of other wider protection schemes, such as the pre-tabular protection method of [16]. In addition, some National Statistical Agencies are questioning current non-perturbative protection methods because "the task of balancing confidentiality and usability [...] is nearly impossible" [27]. Therefore there is a need for new methods, and this justifies the research on CTA and other approaches. Indeed, there is no actually any protection method that fits the needs of all NSAs in the world.

For real and large tables the MILO formulation of CTA results in a difficult optimization problem. It is worth noting that even the linear optimization (LO) problems obtained from large CTA instances by fixing the binary variables are very difficult for today state-of-theart solvers. Indeed, some of these instances have been included in standard LO repositories [24]. Some heuristic approaches have been developed to compute feasible, good suboptimal solutions to the MILO-CTA problem with few computational resources. For instance a block coordinate-descent approach was developed in [17. In [3] this approach was combined with a fix-and-relax heuristic. It should be stressed that "in practice, tabular data protection is the last stage of the "data cycle," and, in an attempt to meet publication deadlines, NSAs require methods that find fast solutions to protect large tables" [11]. For instance, recent online table generation services require real-time (i.e., a few seconds) protection procedures. Since optimization based procedures can not satisfy these low computational times for large tables (where large could mean millions of cells), some NSAs have devised specific methods relying on fast statistical procedures (in short, some noise is added to microdata, and tables are generated from these perturbed microdata) 25]. However those microdata-perturbing approaches ignore the linear constraints of the table, thus protected tables may present inconsistencies.

This work describes a new CTA variant, where the binary variables for lower or upper protection of each sensitive cell are a priori fixed. Rules for fixing the binary decisions will be presented in Section 5 . The resulting continuous problem thus only focuses on minimizing the norm of the cell deviations vector. To guarantee the quality of the protected table, cell deviations are constrained to a tight percentage of the cell value, which, together with the fixed values of the binary variables, may lead to an infeasible solution. To guarantee feasibility we may change the right-hand-side of table relations, the bounds on deviations, or the protection levels. These three criteria, plus the minimization of the norm of the vector of deviations, lead to four different and opposite objectives, resulting in a four-objective optimization problem. This problem will be solved by lexicographic optimization. This is justified by two arguments. First, finding all the efficient solutions by a weighted sum scalarization may involve the solution of a large number of LO-CTA problems, which are known to be very challenging. For example, one of the two instances from the standard LO repository [24] that the recently released Google Glop LO solver could not solve [2] is actually a LO-CTA instance. Second, in this particular application there is a natural hierarchy on the importance of objectives, which justifies the use of lexicographic optimization. This multiobjective approach has been recently implemented within the FP7-INFRA-2010-262608 project funded by the European Union, with the participation, among others, of the NSAs of Germany, Netherlands, Finland, Sweden and Slovenia. The multiobjective LO-CTA software has been included in the $\tau$-Argus package since version 4.1.0 [13, 19] (http://neon.vb.cbs.nl/casc/tau.htm), used by many 
European national statistical institutes for the protection of tabular data.

The quality of the solution provided by the LO-CTA approach will in general be worse than that of the MILO-CTA formulation. However, it will give end users a chance to solve very large instances in moderate time. Other variants have been devised for the same purpose. For instance the CTA variant of [18] solves a sequence of LO problems by randomly assigning a value to some sensitive cells within its protection interval, and trying to adjust the rest of cells with minimum changes. Our multiobjective approach exhibits many differences with that one: (i) it solves only up to four LO problems; (ii) it allows changes in the bounds and constraints (not only on bounds); (iii) and most important, the values of sensitive cells usually guarantee the protection levels when this is the first criteria in the lexicographic order (this will be the case in all the computational experiments performed, since loose constraints were imposed for the other three criteria). If protection levels are not the first criteria in the lexicographic order, small variations in the protections may appear; but this can be acceptable, since protection intervals are anyway heuristically adjusted by data protectors. Alternative approaches, such as the one of [18], do not even pay attention to this fact, and just replace the sensitive cell by a value which is always within the protection interval, thus not safe.

The paper is organized as follows. Section 2 outlines the MILO-CTA model and it presents the LO-CTA problem without binary variables. Sections 3 and 4 introduce the multiobjective LO-CTA model, and how it was solved by lexicographic optimization. Section 5 describes the different procedures used to a priori fix the binary variables. Finally Section 6 provides extensive computational results in the solution of real-world tables using commercial and open source LO solvers; a comparison between LO-CTA and MILO-CTA is also reported, in terms of CPU time and quality of solutions.

\section{Formulation of MILO-CTA and LO-CTA by fixing the binaries}

Any CTA instance can be represented by the following parameters: (i) A set of cell values $a_{i}, i \in \mathcal{N}=\{1, \ldots, n\}$, that satisfy $\mathcal{M}=\{1, \ldots, m\}$ linear relations $A a=b, a$ being the vector of $a_{i}$ 's, $A \in \mathbb{R}^{m \times n}$ and $b \in \mathbb{R}^{m}$. (From now on, we will indistinctly refer to $a_{i}$ as either "cell $i$ " or "cell value $i$ ".) The particular structure of the table is defined by equations $A a=b$. Each tabular constraint imposes that the inner cells have to be equal to the total or marginal cell, i.e., $\left(\sum_{i \in \mathcal{I}_{j}} a_{i}\right)-a_{t_{j}}=0$, where $\mathcal{I}_{j}$ is the set of inner cells of relation $j \in \mathcal{M}$, and $t_{j}$ is the index of the total cell of relation $j$. Any type of table can be modeled by these equations. (ii) A lower and upper bound for each cell $i \in \mathcal{N}$, respectively $l_{a_{i}}$ and $u_{a_{i}}$, which are considered to be known by any attacker. If no previous knowledge is assumed for cell $i, l_{a_{i}}=0\left(l_{a_{i}}=-\infty\right.$ if $a \geq 0$ is not required $)$ and $u_{a_{i}}=+\infty$ can be used. The quality of the protected table can be forced by imposing tight cell bounds, although this may result in infeasibility issues. (iii) A set $\mathcal{S}=\left\{i_{1}, i_{2}, \ldots, i_{s}\right\} \subseteq \mathcal{N}$ of indices of confidential cells. (iv) Nonnegative lower and upper protection levels for each confidential cell $i \in \mathcal{S}$, respectively $l p l_{i}$ and $u p l_{i}$, such that the released values satisfy either $x_{i} \geq a_{i}+u p l_{i}$ or $x_{i} \leq a_{i}-l p l_{i}$. (v) Nonnegative cell weights $w_{i}, i \in \mathcal{N}$, used in the definition of the objective function. These weights penalize perturbations from the original cell values in the released table.

CTA attempts to find the values $x_{i}, i \in \mathcal{N}$, which are closest to $a_{i}$ according to some distance $\ell$, that make the released table safe. The problem can be formulated in terms of 
cell deviations by defining $z=x-a$, thus obtaining:

$$
\begin{array}{ll}
\min _{z} & \|z\|_{\ell} \\
\text { s. to } & A z=0 \\
& l_{a}-a \leq z \leq u_{a}-a \\
& z_{i} \leq-\overline{l p} l_{i} \text { or } z_{i} \geq u p l_{i} \quad i \in \mathcal{S}
\end{array}
$$

$z \in \mathbb{R}^{n}$ being the vector of deviations.

Defining $z=z^{+}-z^{-}, z^{+} \in \mathbb{R}^{n}$ and $z^{-} \in \mathbb{R}^{n}$ being the vector of positive and negative deviations in absolute value, and introducing a vector of binary variables $y \in \mathbb{R}^{s}$ to model the disjunctive constraints (either "upper protection" $z_{i} \geq u p l_{i}$ when $y_{i}=1$ or "lower protection" $z_{i} \leq-l p l_{i}$ when $\left.y_{i}=0\right)$, (1) can be written for the $\ell_{1}$ distance as the following MILO problem:

$$
\begin{aligned}
\min _{z^{+}, z^{-}, y} & \sum_{i \in \mathcal{N}} w_{i}\left(z_{i}^{+}+z_{i}^{-}\right) \\
\text {s. to } & A\left(z^{+}-z^{-}\right)=0 \\
& u p l_{i} y_{i} \leq z_{i}^{+} \leq\left(u_{a_{i}}-a_{i}\right) y_{i} \quad i \in \mathcal{S} \\
& l p l_{i}\left(1-y_{i}\right) \leq z_{i}^{-} \leq\left(a_{i}-l_{a_{i}}\right)\left(1-y_{i}\right) \quad i \in \mathcal{S} \\
& 0 \leq z^{+} \leq u_{a}-a \\
& 0 \leq z^{-} \leq a-l_{a} \\
& y_{i} \in\{0,1\} \quad i \in \mathcal{S} .
\end{aligned}
$$

When $y_{i}=1$ the constraints mean $u p l_{i} \leq z_{i}^{+} \leq\left(u_{a_{i}}-a_{i}\right)$ and $z_{i}^{-}=0$, thus the protection direction is "upper"; when $y_{i}=0$ we get $z_{i}^{+}=0$ and $l p l_{i} \leq z_{i}^{-} \leq\left(a_{i}-l_{a_{i}}\right)$, thus protection direction is "lower". Model (2) is a difficult MILO problem for medium-large instances.

For large tables the MILO-CTA model is impractical if a quick solution is required. In those situations, an efficient alternative would be to a priori fix the binary variables, thus obtaining a continuous LO-CTA formulation. Possible infeasibilities in the resulting problem could be dealt with the approaches exposed in [10], some of them already used in the context of CTA [8]. Fixing in (2) the binary variables, the continuous $\ell_{1}$-CTA approach can be formulated as the following LO problem:

$$
\begin{array}{cc}
\min _{z^{+}, z^{-}} & \sum_{i \in \mathcal{N}} w_{i}\left(z_{i}^{+}+z_{i}^{-}\right) \\
\text {s. to } & A\left(z^{+}-z^{-}\right)=0 \\
& l^{+} \leq z^{+} \leq u^{+} \\
& l^{-} \leq z^{-} \leq u^{-}
\end{array}
$$


where $l^{+}, l^{-}, u^{+}, u^{-} \in \mathbb{R}^{n}$ are defined as

$$
\begin{gathered}
l_{i}^{+}= \begin{cases}u p l_{i} & \text { if } i \in \mathcal{S} \text { and } y_{i}=1 \\
0 & \text { if }(i \in \mathcal{N} \backslash \mathcal{S}) \text { or }\left(i \in \mathcal{S} \text { and } y_{i}=0\right)\end{cases} \\
u_{i}^{+}= \begin{cases}0 & \text { if } i \in \mathcal{S} \text { and } y_{i}=0 \\
u_{a_{i}}-a_{i} & \text { if }(i \in \mathcal{N} \backslash \mathcal{S}) \text { or }\left(i \in \mathcal{S} \text { and } y_{i}=1\right)\end{cases} \\
l_{i}^{-}= \begin{cases}l p l_{i} & \text { if } i \in \mathcal{S} \text { and } y_{i}=0 \\
0 & \text { if }(i \in \mathcal{N} \backslash \mathcal{S}) \text { or }\left(i \in \mathcal{S} \text { and } y_{i}=1\right)\end{cases} \\
u_{i}^{-}= \begin{cases}0 & \text { if } i \in \mathcal{S} \text { and } y_{i}=1 \\
a_{i}-l_{a_{i}} & \text { if }(i \in \mathcal{N} \backslash \mathcal{S}) \text { or }\left(i \in \mathcal{S} \text { and } y_{i}=0\right) .\end{cases}
\end{gathered}
$$

\section{Guaranteeing a good solution: multiobjective optimization problem}

Fixing binary variables $y \in\{0,1\}^{s}$ in (2) the resulting continuous problem (3) may be infeasible. Infeasibilities can be avoided by modifying the feasible region of (3), i.e., changing the right hand side coefficients and/or the bounds of the variables. We can achieve feasibility by three means:

- Allowing changes in the table relations. Constraints $A z=0$ are transformed to

$$
A z+\alpha^{+}-\alpha^{-}=0, \quad \alpha^{+} \in \mathbb{R}^{m}, \alpha^{-} \in \mathbb{R}^{m},\left(\alpha^{+}, \alpha^{-}\right) \geq 0 .
$$

- Decreasing $l_{a}$ and increasing $u_{a}$, whenever possible. Thus, instead of $l_{a} \leq a \leq u_{a}$ we will consider

$$
l_{a}-\beta_{l} \leq a \leq u_{a}+\beta_{u}, \quad \beta_{l} \in \mathbb{R}^{n}, \beta_{u} \in \mathbb{R}^{n},\left(\beta_{l}, \beta_{u}\right) \geq 0 .
$$

- Reducing $u p l_{i}$ and $l p l_{i}, i \in \mathcal{S}$. Thus, the new lower protection levels will be

$$
l p l:=l p l-\gamma_{l}, \quad \text { upl }:=u p l-\gamma_{u}, \quad \gamma_{l} \in \mathbb{R}^{s}, \gamma_{u} \in \mathbb{R}^{s},\left(\gamma_{l}, \gamma_{u}\right) \geq 0 .
$$

Therefore, instead of the possibly infeasible (3), we can consider the always feasible problem

$$
\begin{array}{cl}
\min _{\omega} & \left(f_{1}\left(z^{+}, z^{-}\right), f_{2}\left(\alpha^{+}, \alpha^{-}\right), f_{3}\left(\beta_{l}, \beta_{u}\right), f_{4}\left(\gamma_{l}, \gamma_{u}\right)\right) \\
\text { s. to } & A\left(z^{+}-z^{-}\right)+\alpha^{+}-\alpha^{-}=0 \\
& l^{+}\left(\gamma_{u}\right) \leq z^{+} \leq u^{+}\left(\beta_{u}\right) \\
& l^{-}\left(\gamma_{l}\right) \leq z^{-} \leq u^{-}\left(\beta_{l}\right) \\
& \left(\alpha^{+}, \alpha^{-}, \beta_{l}, \beta_{u}, \gamma_{l}, \gamma_{u}\right) \geq 0
\end{array}
$$

where

$$
\begin{aligned}
f_{1}\left(z^{+}, z^{-}\right) & =\sum_{i \in \mathcal{N}} w_{i}\left(z_{i}^{+}+z_{i}^{-}\right) \\
f_{2}\left(\alpha^{+}, \alpha^{-}\right) & =e^{\top}\left(\alpha^{+}+\alpha^{-}\right) \\
f_{3}\left(\beta_{l}, \beta_{u}\right) & =e^{\top}\left(\beta_{l}+\beta_{u}\right) \\
f_{4}\left(\gamma_{l}, \gamma_{u}\right) & =e^{\top}\left(\gamma_{l}+\gamma_{u}\right),
\end{aligned}
$$


$e$ is a vector of ones of appropriate length, $\omega=\left(z^{+}, z^{-}, \alpha^{+}, \alpha^{-}, \beta_{l}, \beta_{u}, \gamma_{l}, \gamma_{u}\right)$ denotes the full set of variables of the optimization problem, and

$$
\begin{aligned}
& l_{i}^{+}\left(\gamma_{u_{i}}\right)= \begin{cases}u p l_{i}-\gamma_{u_{i}} & \text { if } i \in \mathcal{S} \text { and } y_{i}=1 \\
0 & \text { if }(i \in \mathcal{N} \backslash \mathcal{S}) \text { or }\left(i \in \mathcal{S} \text { and } y_{i}=0\right)\end{cases} \\
& u_{i}^{+}\left(\beta_{u_{i}}\right)= \begin{cases}0 & \text { if } i \in \mathcal{S} \text { and } y_{i}=0 \\
u_{a_{i}}+\beta_{u_{i}}-a_{i} & \text { if }(i \in \mathcal{N} \backslash \mathcal{S}) \text { or }\left(i \in \mathcal{S} \text { and } y_{i}=1\right)\end{cases} \\
& l_{i}^{-}\left(\gamma_{l_{i}}\right)= \begin{cases}l p l_{i}-\gamma_{l_{i}} & \text { if } i \in \mathcal{S} \text { and } y_{i}=0 \\
0 & \text { if }(i \in \mathcal{N} \backslash \mathcal{S}) \text { or }\left(i \in \mathcal{S} \text { and } y_{i}=1\right)\end{cases} \\
& u_{i}^{-}\left(\beta_{l_{i}}\right)= \begin{cases}0 & \text { if } i \in \mathcal{S} \text { and } y_{i}=1 \\
a_{i}-\left(l_{a_{i}}-\beta_{l_{i}}\right) & \text { if }(i \in \mathcal{N} \backslash \mathcal{S}) \text { or }\left(i \in \mathcal{S} \text { and } y_{i}=0\right) .\end{cases}
\end{aligned}
$$

Abusing of notation, from now on we will use indistinctly $f_{1}\left(z^{+}, z^{-}\right)$and $f_{1}(\omega)$, and similarly for $f_{2}, f_{3}$ and $f_{4}$. The feasible region of (8) will be written in compact form below as $\omega \in \Omega$.

Problem (8) is a multiobjective optimization problem. Its purpose is to obtain a protected table that minimizes $f_{1}$, but at the same time is feasible for the original constraints, i.e., it minimizes $f_{2}, f_{3}$ and $f_{4}$. Note that $\left(f_{1}, f_{2}, f_{3}, f_{4}\right)$ are conflicting objectives: increasing one we can reduce the others. For instance, in the limit, increasing enough $\gamma_{l}$ and $\gamma_{u}$ (i.e., $\gamma_{l}=l p l$ and $\gamma_{u}=u p l$, thus removing the protection levels) makes $z=\beta_{l}=\beta_{u}=0$ and $\alpha^{+}=\alpha^{-}=0$. We also note that upper bounds for $\gamma_{l_{i}}$ and $\gamma_{u_{i}}$ are not needed, although this could result in negative protection levels $u p l_{i}$ and $l p l_{i}$. Indeed this would only happen if $z_{i}^{+}$and $z_{i}^{-}$were negative, which would unnecessarily increase the objective function; therefore, in an optimal solution such situation is not possible.

\section{Solving the multiobjective optimization problem}

In general, in a multiobjective optimization problem there is no solution $\omega^{*}$ that minimizes all the objective functions $f_{i}, i=1, \ldots, p$ simultaneously ( $p$ being the number of objectives, $p=4$ in our problem). The goal is thus to obtain a Pareto optimal solution (also named efficient or nondominated solution). A feasible point $\omega^{*}$ is named Pareto optimal or efficient solution if there is no point that dominates it, where, in a minimization problem with $p$ objectives, it is said that $\omega^{1}$ dominates $\omega^{2}$ if $f_{i}\left(\omega^{1}\right) \leq f_{i}\left(\omega^{2}\right), i=1, \ldots, p$, and, for some $j \in\{1, \ldots, p\}, f_{j}\left(\omega^{1}\right)<f_{j}\left(\omega^{2}\right)$. Therefore, Pareto optimal or efficient solutions cannot improve any of the objectives without deteriorating some of the other ones. Two related concepts are those of weakly efficient and strictly efficient solutions:

- A feasible point $\omega^{*}$ is named weakly Pareto optimal or weakly efficient if there is no point $\omega \in \Omega$ such that $f_{i}(\omega)<f_{i}\left(\omega^{*}\right)$ for $i=1, \ldots, p$.

- A feasible point $\omega^{*}$ is named strictly Pareto optimal or strictly efficient if there is no point $\omega \in \Omega$ such that $f_{i}(\omega) \leq f_{i}\left(\omega^{*}\right)$ for $i=1, \ldots, p$.

From the above definitions, it is clear that strict efficiency implies efficiency, and efficiency implies weak efficiency.

There is a fourth type of solution, named properly efficient solution. These solutions are generated by the first solution approach (namely, scalarization) of the two outlined in Subsection 4.1. Although we will not use this approach, but the second one (namely, lexicographic optimization), we briefly overview properly efficiency for two reasons: (i) in case scalarization is considered in the future to solve the multiobjective CTA problem; (ii) 
to show that in the multiobjective LO-CTA problem efficient solutions are also properly efficient.

Properly efficient solutions guarantee that trade-offs between objectives are bounded. Formally, a feasible point $\omega^{*} \in \Omega$ is properly efficient in Geoffrion sense [15] if it is efficient and there is a real number $M>0$ such that for all $i=1, \ldots, p$, and $\omega \in \Omega$ satisfying $f_{i}(\omega)<f_{i}\left(\omega^{*}\right)$ there exists an index $j$ with $f_{j}\left(\omega^{*}\right)<f_{j}(\omega)$ such that

$$
\frac{f_{i}\left(\omega^{*}\right)-f_{i}(\omega)}{f_{j}(\omega)-f_{j}\left(\omega^{*}\right)} \leq M .
$$

An alternative definition of properly efficiency was provided by Kuhn and Tucker [22. Formulating the multiobjective optimization problem as

$$
\begin{aligned}
& \min f(\omega) \\
& \text { s. to } \omega \in \Omega=\{\omega: g(\omega) \leq 0\}
\end{aligned}
$$

where $f: \mathbb{R}^{n^{\prime}} \rightarrow \mathbb{R}^{p}$ and $g: \mathbb{R}^{n^{\prime}} \rightarrow \mathbb{R}^{m^{\prime}}$, a feasible point $\omega^{*} \in \Omega$ is properly efficient in Kuhn and Tucker sense if it is efficient and there is no $d$ satisfying:

$$
\begin{aligned}
& \nabla f_{j}\left(\omega^{*}\right)^{\top} d \leq 0 \forall j=1, \ldots, p \\
& \nabla f_{i}\left(\omega^{*}\right)^{\top} d<0 \text { for some } i \in\{1, \ldots, p\} \\
& \nabla g_{k}\left(\omega^{*}\right)^{\top} d \leq 0 \forall k \in \mathcal{A}\left(\omega^{*}\right)=\left\{k \in\{1, \ldots, m\}: g_{k}\left(\omega^{*}\right)=0\right\}
\end{aligned}
$$

Conditions (13) mean that there is not a direction $d$ from $\omega^{*}$ such that no objective locally increases, at least one strictly locally decreases, and all the points along this direction remain locally feasible. It is known that if $f$ and $g$ in $(12)$ are convex and continuously differentiable, then if $\omega^{*}$ is properly efficient in Kuhn and Tucker sense, it is properly efficient in Geoffrion sense. We can now show that in the multiobjective LO-CTA problem all efficient solutions are properly efficient:

Proposition 1 All the efficient solutions of the multiobjective LO-CTA problem (8) are properly efficient (in Kuhn and Tucker sense, and then in Geoffrion sense).

Proof. Problem (8) can be transformed to the form (12) (equalities $A z+\alpha^{+}-\alpha^{-}=0$ can be written as $A z+\alpha^{+}-\alpha^{-} \leq 0$ and $\left.-A z-\alpha^{+}+\alpha^{-} \leq 0\right)$, the feasible set $\Omega$ being a polyhedron. By (9), the four objectives $f_{i}, i=1, \ldots, 4$, are linear and bounded below by 0 in the feasible region (since they are $\ell_{1}$ norms). Since it is a linear problem, any direction $d$ satisfying conditions (13) would be an unboundness direction for some objective $i \in\{1, \ldots, 4\}$, which is a contradiction.

\subsection{Solution approaches}

We outline two of the most used approaches for multiobjective optimization, scalarization and lexicographic optimization:

- In the scalarization approach the vectorial or multiobjective function $\left(f_{1}, \ldots, f_{p}\right)$ can be transformed to a scalar function $f$ by using a linear convex combination of the components, i.e:

$$
f=\sum_{i=1}^{p} \lambda_{i} f_{i} \quad \lambda_{i} \geq 0 \quad i=1, \ldots, p, \quad \sum_{i=1}^{p} \lambda_{i}=1 .
$$


1. Let $\pi(j)$ be the preference order of $f_{j}, j=1, \ldots, p$.

2. for $j=1 \ldots p$ do

3. Solve

$$
\begin{gathered}
f_{\pi(j)}^{*}=\min _{\omega} f_{\pi(j)}(\omega) \\
\text { s. to } f_{\pi(l)}(\omega) \leq f_{\pi(l)}^{*} \quad l=1, \ldots, j-1 \\
\omega \in \Omega .
\end{gathered}
$$

4. end for

5. $\operatorname{return}\left(\omega^{*}\right.$, solution of last optimization problem solved)

Figure 3.: The lexicographic optimization approach for (8)

The particular weights $\lambda_{i}, i=1, \ldots, 4$ should be provided by the user. Let $\omega^{*}$ be a solution to

$$
\min f(\omega) \text { s.to } \omega \in \Omega
$$

and $\lambda=\left(\lambda_{1}, \ldots, \lambda_{p}\right)$. It is known that (i) if $\lambda>0$ then $\omega^{*}$ is a properly efficient solution; (ii) if $\lambda \geq 0$ then $\omega^{*}$ is a weakly efficient solution; (iii) if $\lambda \geq 0$ and $\omega^{*}$ is the unique solution of $(16)$ then $\omega^{*}$ is a strictly efficient solution (note this would hold in the multiobjective $\ell_{2}$-CTA problem, since the objective function of (16) would be strictly convex). In addition, all the properly and weakly efficient solutions can be obtained from some suitable $\lambda$.

In this approach is important to scale the functions $f_{i}$ to guarantee that their different units can be added to a single $f$. We could use as scaling factor for $f_{i}$ the value $\bar{f}_{i}=\max \left\{\left|f_{i}^{*}\right|, 1\right\}$, where $f_{i}^{*}$ is the optimal value for this function (i.e., as if we solved the multiobjective problem considering $\lambda_{i}=1$ and $\lambda_{j}=0, j \neq i$ ). However, in practice, $f_{i}^{*}$ is unknown (unless we solve the optimization problem using $f_{i}$ as the single objective), and then some other value $f_{i}^{0}=f_{i}\left(\omega^{0}\right)$ at some (initial) feasible point $\omega^{0}$ may be used. Therefore the objective function to be used in practice could be

$$
f=\sum_{i=1}^{p} \lambda_{i} \frac{f_{i}}{\bar{f}_{i}} \quad \lambda_{i} \geq 0 \quad i=1, \ldots, p, \quad \sum_{i=1}^{p} \lambda_{i}=1 .
$$

- Lexicographic optimization or lexmin optimization considers a preference order for the different objectives, given by the permutation $\pi:\{1, \ldots, p\} \rightarrow\{1, \ldots, p\}, \pi(1)$ and $\pi(p)$ being respectively the objectives with highest and lowest preference. The algorithm is shown in Figure 3. This is the approach adopted in this work, since problem (8) exhibits a natural ordering of the objectives. In particular, preference should be given to feasible solutions (i.e., solutions where $\alpha^{+}, \alpha^{-}, \beta_{l}, \beta_{u}, \gamma_{l}$, and $\gamma_{u}$ are zero), and therefore in general $\pi(4)=1$ (i.e., $f_{1}(z)=\|z\|_{\ell}$ has the lowest preference). Of the remaining three objectives in $(9), f_{4}$ should in general have the highest preference, since it controls the sensitive cells protection levels, so $\pi(1)=4$. In this case, if no tight bounds are considered for $\alpha^{+}, \alpha^{-}, \beta_{l}$ and $\beta_{u}$-as it was the case in all the computational tests performed $-\gamma_{l}$ and $\gamma_{u}$ will be 0 , thus the first optimization problem is trivially solved, and protection levels are never perturbed. This will be clearly observed in the computational results section. The preference order for $f_{2}, f_{3}$ (that is, to give more priority to bounds or right-hand-side changes) should be decided by the user; different preferences will result in different solutions. Any solution to this lexmin problem is a Pareto or efficient solution. Given a particular preference order $\pi$, the algorithm of Figure 3 will provide a Pareto or efficient solution to (8). 


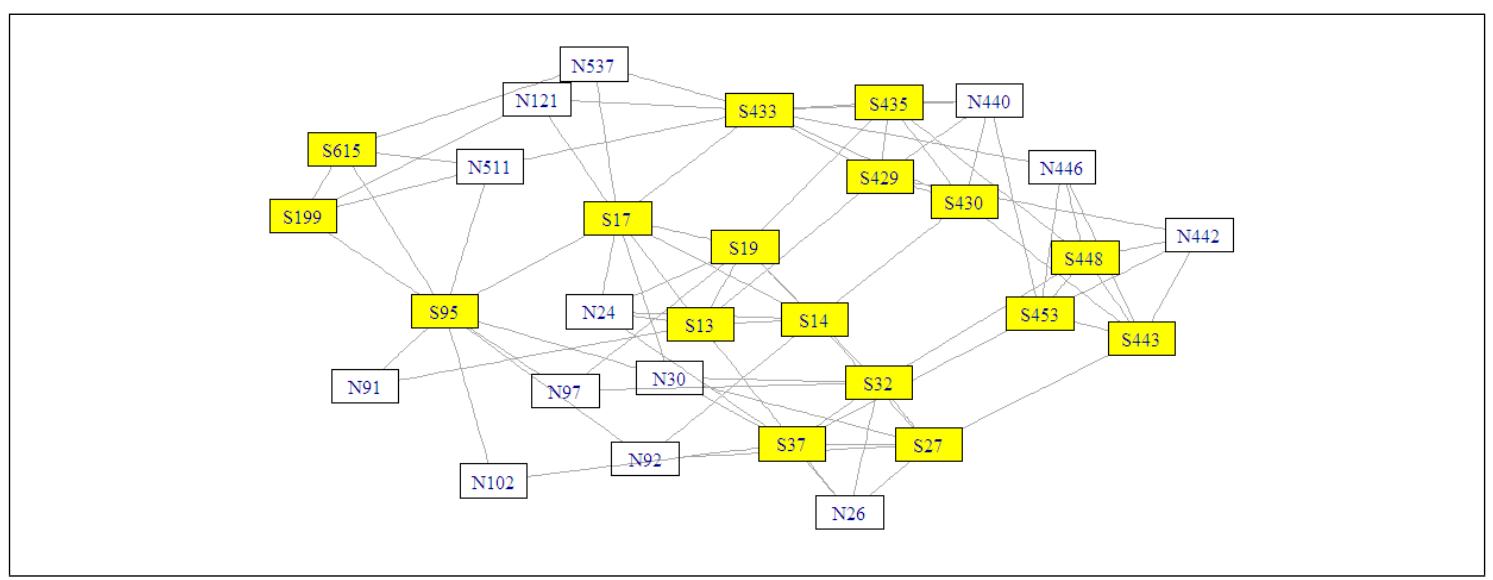

Figure 4.: The graph obtained by the network approach for instance "table7".

Given the natural ordering of the objectives, in this work we have considered lexicographic optimization. However this approach requires the solution of up to four LO problems, the last one being a challenging one, while scalarization would only involve one LO problem.

\section{Fixing the binary variables}

A good assignment of the binary variables is instrumental both for the feasibility and the quality of the solution provided by the lexmin approach. However, there is no direct method for an a priori good and feasible assignment for any CTA instance. Hopefully, any approximate approach should provide a good solution or, at least, to avoid solutions with severe infeasibilities in the resulting LO models.

The simplest approach is random assignment. Sensitive cells are oriented either to positive or negative directions with equal probabilities. Although randomness is fairly good at dividing the sensitive cells between both directions, this method cannot take into account any specific feature of the table.

The SAT heuristic (which was developed in [17] for the solution of the MILO-CTA problem by a block coordinate descent heuristic) can be useful to obtain a wiser proposal. Directions of sensitive cells are computed by solving a satisfiability $(S A T)$ problem on rules derived from the table constraints (see [17] for details). Nevertheless, some cells could be undetermined if no rule is indicating a given direction, and these cells should be assigned at random. The SAT approach (as the below network and both SAT-network approaches) cannot guarantee a feasible solution of (2). However they help in reducing the level of infeasibility, compared to the previously discussed random assignment, such that components $f_{2}, f_{3}$ and $f_{4}$ of $(8)$ will have a lower value in the optimal solution.

As for the SAT approach, the next new alternative developed in this work is based also in the constraints structure of the problem, and neither guarantees a feasible solution. This method is called network because a graph is constructed from the set of constraints of the CTA problem. An example of such a graph can be seen in Figure 4 , obtained from the real instance "table7", one of the test cases from Table 1 to be used in the computational results of Section 6. In this graph the vertices represent cells and the edges constraints. Specifically, the graph is built in two steps:

(1) First, we create a vertex for every sensitive cell, and an edge between every pair of vertices such that there is a constraint containing both cells.

(2) Second, we add a vertex for every non-sensitive cell appearing at least in two constraints together with a sensitive cell. 
In Figure 4, sensitive and non-sensitive cells have been represented by yellow and white boxes, with labels started by "S" and "N", respectively. In both cases, the number in the label corresponds to the cell number for this particular instance.

Each vertex may have a direction +1 or -1 , and we start with a graph where all the vertices are undefined (say, 0 ). The goal is to assign a direction to each vertex so that the number of mismatches is the minimum. In a broad sense, we call mismatch a situation where both nodes of some edge are contributing to the constraint imbalance in the same direction. For better understanding, consider the structure of general constraints of a table:

$$
A z=0 \Leftrightarrow a_{i, j_{1}} z_{j_{1}}+a_{i, j_{2}} z_{j_{2}}+\cdots+a_{i, j_{k}} z_{j_{k}}=0 \quad i \in \mathcal{M}
$$

Usually, only a few cells are involved in the $i$-th constraint (say, $k$ cells), such that $k-1$ coefficients are 1 and just one is -1 , meaning that the sum of deviations $z$ must be equal to the marginal deviation. So, two cells linked by an edge are matched when:

- both point upwards and one of them has a negative coefficient;

- both point downwards and one of them has a negative coefficient;

- they point to different directions and have positive coefficients.

The main algorithm of the network method attempts to find the most suitable orientation to each sensitive cell, trying to match as many edges as possible. So, starting from a given vertex, it traverses the graph in an order defined from its topology (depending on the first vertex, the order may be different, so the assignment would differ as well).

Since a cell may appear in several constraints, conflicting situations appear often, as long as any assignment to the current vertex would cause a mismatch in some of the incident edges. In such situations the heuristic implemented chooses the direction that minimizes the number of mismatches, or a random choice in case of a tie.

Finally, the heuristic repeats the main algorithm from many starting points, computing a global index of mismatch for the assignment. The procedure returns the assignment with the least index.

A hybrid between the SAT and network methods is also possible: the suggested directions given by the SAT output are fixed and not assigned within the network process. This fourth approach will be named both SAT-network.

The above four approaches (random, SAT, network and both SAT-network) to fix the protection directions of sensitive cells are evaluated in Section 6.4.

\section{Computational results}

The lexicographic approach based on LO-CTA has been implemented within the FP7INFRA-2010-262608 "Data without Boundaries" European Union project. This code has been included in the $\tau$-Argus package since version 4.1.0 [13, 19] (which can be freely obtained from http://neon.vb.cbs.nl/casc/tau.htm), used by European (and some non-European) statistical agencies. The code may use up to five different solvers, both commercial and open source. In this work we will only provide results with the commercial solvers CPLEX and XPRESS, and the open source solver Clp [14] from the COIN-OR project. All the runs were carried out on a Fujitsu Primergy RX300 server with two $3.33 \mathrm{GHz}$ Intel Xeon X5680 CPUs (each CPU with 12 cores) and 144 GB of RAM, under a GNU/Linux operating system (Suse 11.4), without exploitation of multithreading capabilities.

We have considered a set of 38 standard instances in the literature on statistical disclosure control on tabular data [4, 6, 18. Most of them are publicly available real tables 


\begin{tabular}{|c|c|c|c|c|}
\hline instance & $n$ & $s$ & $m$ & n. coef \\
\hline australia $\mathrm{ABS}$ & 24420 & 918 & 274 & 13224 \\
\hline bts4 & 36570 & 2260 & 36310 & 136912 \\
\hline cbs & 11163 & 2467 & 244 & 22326 \\
\hline dale & 16514 & 4923 & 405 & 33028 \\
\hline destatis & 5940 & 621 & 1464 & 18180 \\
\hline five $20 b$ & 34552 & 3662 & 52983 & 208335 \\
\hline five20c & 34501 & 4022 & 58825 & 231345 \\
\hline hier13 & 2020 & 112 & 3313 & 11929 \\
\hline hier13x13x13a & 2197 & 108 & 3549 & 11661 \\
\hline hier $13 \times 13 \times 13 b$ & 2197 & 108 & 3549 & 11661 \\
\hline hier $13 \times 13 \times 13 c$ & 2197 & 108 & 3549 & 11661 \\
\hline hier $13 \times 13 \times 13 d$ & 2197 & 108 & 3549 & 11661 \\
\hline hier $13 \times 13 \times 13 e$ & 2197 & 112 & 3549 & 11661 \\
\hline hier $13 \times 13 \times 7 d$ & 1183 & 75 & 1443 & 5369 \\
\hline hier $13 \times 7 \times 7 d$ & 637 & 50 & 525 & 2401 \\
\hline hier16 & 3564 & 224 & 5484 & 19996 \\
\hline hier16x16x16a & 4096 & 224 & 5376 & 21504 \\
\hline hier $16 \times 16 \times 16 b$ & 4096 & 224 & 5376 & 21504 \\
\hline hier $16 \times 16 \times 16 \mathrm{c}$ & 4096 & 224 & 5376 & 21504 \\
\hline hier16x16x16d & 4096 & 224 & 5376 & 21504 \\
\hline hier $16 \times 16 \times 16 \mathrm{e}$ & 4096 & 224 & 5376 & 21504 \\
\hline nine12 & 10399 & 1178 & 11362 & 52624 \\
\hline nine $5 d$ & 10733 & 1661 & 17295 & 58135 \\
\hline ninenew & 6546 & 858 & 7340 & 32920 \\
\hline osorio & 10201 & 7 & 202 & 20402 \\
\hline sbs2008 C & 4212 & 1135 & 2580 & 13806 \\
\hline sbs2008_D_b & 28288 & 7131 & 13360 & 87022 \\
\hline $\operatorname{sbs} 2008^{-} \mathrm{E}$ & 1430 & 382 & 991 & 4680 \\
\hline table1 & 1584 & 146 & 510 & 4752 \\
\hline table3 & 4992 & 517 & 2464 & 19968 \\
\hline table4 & 4992 & 517 & 2464 & 19968 \\
\hline table5 & 4992 & 517 & 2464 & 19968 \\
\hline table6 & 1584 & 146 & 510 & 4752 \\
\hline table7 & 624 & 17 & 230 & 1872 \\
\hline table8 & 1271 & 3 & 72 & 2542 \\
\hline targus & 162 & 13 & 63 & 360 \\
\hline toy3dsarah & 2890 & 376 & 1649 & 9690 \\
\hline two5in6 & 5681 & 720 & 9629 & 34310 \\
\hline
\end{tabular}

Table 1. Dimensions of instances.

generated by NSAs, and only a few of them are confidential tables generated by Eurostat (instances "sbs*") - the statistical agency of the European Commission, and Destatis (instance "destatis") - the German NSA. Table 1 reports the main characteristics of these instances: number of cells (column $n$ ), number of sensitive cells (column $s$ ), number of constraints (column $m$ ), and number of nonzero coefficients in the constraints matrix $A$ defining the table relations.

To avoid errors due to numerical tolerances, in the implementation developed constraints $f_{\pi(l)}(\omega) \leq f_{\pi(l)}^{*}$ of 114$)$ were replaced by $f_{\pi(l)}(\omega) \leq f_{\pi(l)}^{*}\left(1+\epsilon_{f}\right), \epsilon_{f}$ being a small value (e.g., $\left.10^{-4}\right)$. Using a value $\epsilon_{f}=0$ resulted in larger solution times, and, very often, in infeasibility issues.

The first four following subsections report the results obtained in the evaluation of priority orders, solvers, percentages of deviations in cell values, and procedures for fixing the protection directions of sensitive cells with LO-CTA. The fifth subsection provides a comparison between LO-CTA and MILO-CTA in terms of solution time and quality of the solution.

\subsection{Evaluation of priority orders}

Table 2 shows the results for the evaluation of priority orders $\pi=(4,3,2,1)$ and $\pi=$ $(4,2,3,1)$ (where $\pi=(\pi(1), \ldots, \pi(4))$. For each instance the table reports the CPU time of the lexmin optimization, and the optimal value of each objective function (columns $\left.f_{i}, i=1, \ldots, 4\right)$. The fastest run is marked in boldface. These executions were performed with the standard (i.e., the infeasible primal-dual path-following) barrier algorithm of CPLEX, randomly fixing the protection directions of sensitive cells (but using the same 


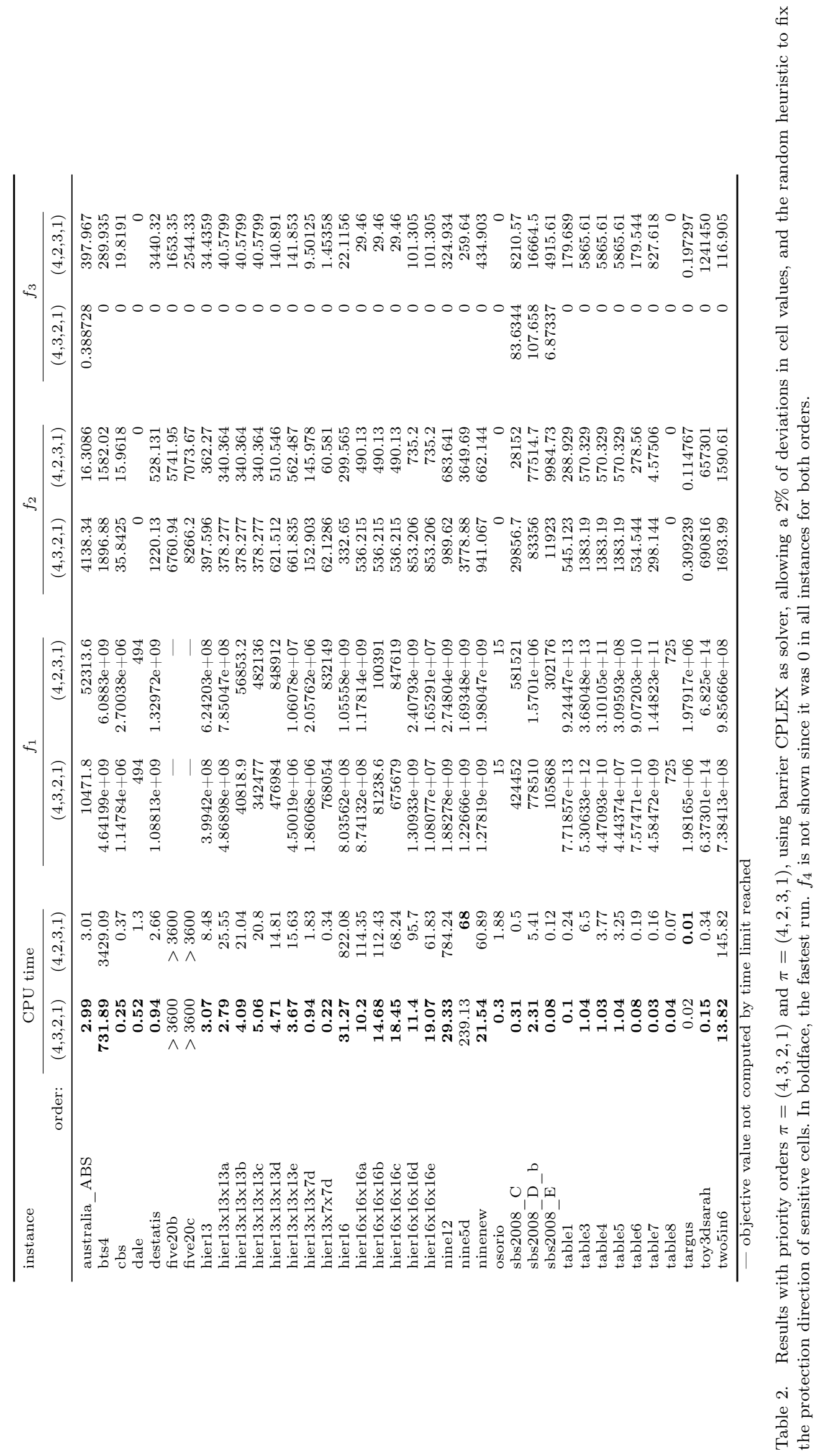




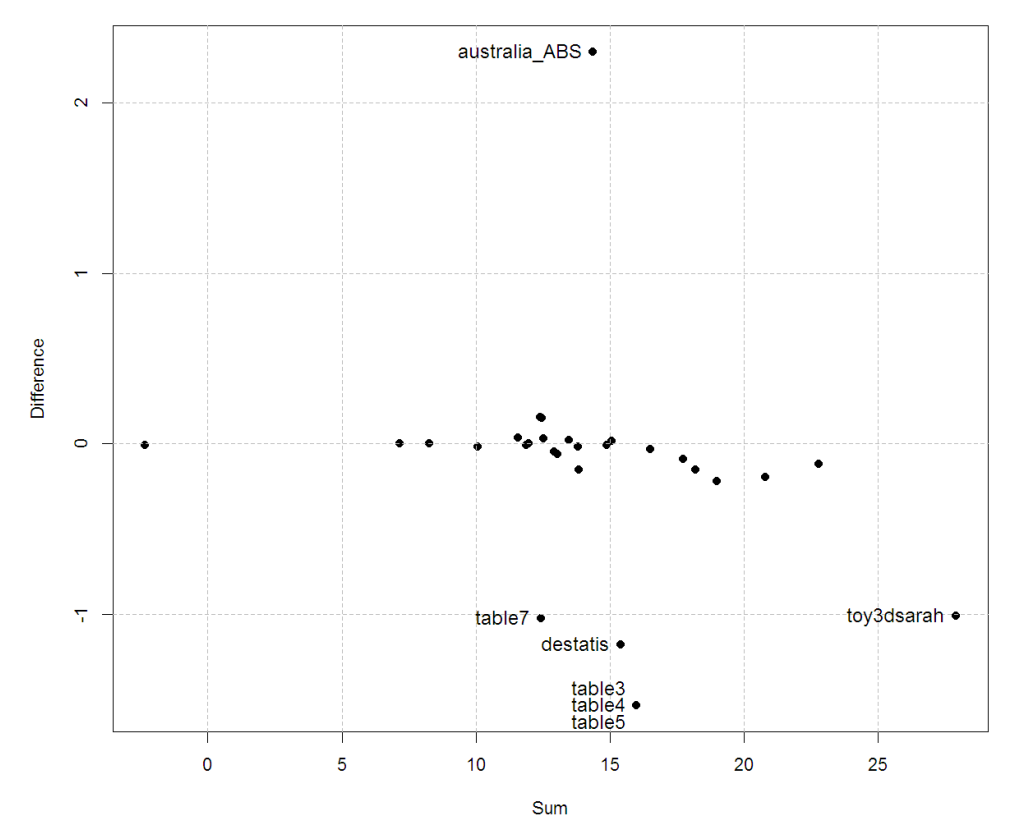

Figure 5.: Plot of Sum $=\log \left(f_{2}^{(4,3,2,1)}+f_{3}^{(4,3,2,1)}\right)+\log \left(f_{2}^{(4,2,3,1)}+f_{3}^{(4,2,3,1)}\right)$ vs Difference $=\log \left(f_{2}^{(4,3,2,1)}+f_{3}^{(4,3,2,1)}\right)-\log \left(f_{2}^{(4,2,3,1)}+f_{3}^{(4,2,3,1)}\right)$, for every instance.

random directions for the two priority orders), and allowing a $2 \%$ of deviations in the cells values. This $2 \%$ maximum deviation in cell values is a practical requirement of NSAs (in particular it was told to us by Destatis, the German NSA), in a attempt to maximize the utility of the resulting table, making the optimization problem significantly more difficult. The time limit of 3600 was reached for two instances, whose value for $f_{1}$ is marked with "--" (the other problems could be solved within the time limit).

Objective $f_{4}$ is not shown since it was 0 in all instances for both orders (as it was discussed in Subsection 4.1). This means (quite obviously) that we do not need to change the protection levels if bounds and constraints can be modified. For order $\pi=(4,3,2,1)$ in all the instances, but four, bounds were not modified (that is, $f_{3}=0$ ), which forced significant changes in constraints (that is, $f_{2}$ is large). For order $\pi=(4,2,3,1)$ both $f_{2}$ and $f_{3}$ were positive, but the changes in constraints were not so large. If we focus on the norm of the cell deviations, $f_{1}$ was much less for $\pi=(4,3,2,1)$, and this also happened for the CPU time (only in two instances the order $\pi=(4,2,3,1)$ provided a fastest execution). At a first glance, it seems there is no clear winner: on the one hand, according to $f_{1}$ and the CPU time, it could be concluded that $\pi=(4,3,2,1)$ is preferable; on the other hand, this order involves larger modifications in constraints due to $f_{2}$. Both orders provide efficient or Pareto solutions, and depending on their different properties, NSAs may prefer one order or the other. Figure 5 gives us more insight into the behaviour of both orders. The horizontal axis of that figure is related to the sum $\log \left(f_{2}^{(4,3,2,1)}+f_{3}^{(4,3,2,1)}\right)+\log \left(f_{2}^{(4,2,3,1)}+f_{3}^{(4,2,3,1)}\right)$, while the vertical axis is associated with the difference $\log \left(f_{2}^{(4,3,2,1)}+f_{3}^{(4,3,2,1)}\right)-\log \left(f_{2}^{(4,2,3,1)}+f_{3}^{(4,2,3,1)}\right)$; each instance is represented by a point in the figure. Note that it makes sense to consider $f_{2}+f_{3}$, since $f_{2}$ represents changes in constraints (that is, deviations from the value of the marginal cell, since nonzero values in matrix $A$ are only +1 or -1 ), and $f_{3}$ changes in cell bounds. From the figure, we see that the differences are around zero for most instances, that is, the amount of constraints and bounds changes $f_{2}+f_{3}$ was similar for both orders. The only 
exceptions were "australia_ABS" (in which order $\pi=(4,3,2,1)$ provided a larger $f_{2}+f_{3}$ ), and "destatis", "toy3dsarah", "table3", "table4", "table5" and "table7" (order $\pi=(4,2,3,1)$ provided the larger changes in those cases). Therefore, from Figure 5 it cannot be stated that the less CPU time and value of $f_{1}$ provided by $\pi=(4,3,2,1)$ are due to this order providing larger changes. According to this, order $\pi=(4,3,2,1)$ could be considered a better choice.

For the computational results of the following Subsections 6.2 6.4 we will consider the order $\pi=(4,2,3,1)$ : being more time consuming, it can be considered the worst case, thus the preferred option for a fair evaluation of LO-CTA. Both priority orders will be again considered in Subsection 6.5, in which LO-CTA is compared to MILO-CTA.

\subsection{Evaluation of solvers}

Table 3 shows the CPU time with the interior-point (columns "barrier") and simplex algorithms of the commercial solvers CPLEX and XPRESS, and the open source Clp solver from the COIN-OR project. These executions were performed with priority order $\pi=(4,2,3,1)$, allowing a $2 \%$ of deviations in cell values, and the random heuristic to fix the protection direction of sensitive cells. The CPU time of the fastest algorithm (interior-point or simplex) for each solver is marked in italics; in addition, the fastest run among all the solvers is in boldface. Problems marked with "-" for Clp were reported as infeasible (though it is feasible). The time limit of 3600 seconds was reached for several instances, thus no efficient solution was computed in those situations.

The immediate conclusion from the results of Table 3 is that interior-point algorithms are more efficient than simplex in this kind of application, mainly for the two commercial solvers (it is known than the interior-point of Clp is not competitive against commercial implementations, unlike its simplex code which is highly efficient). This is consistent with previous works on CTA [4, 6, 18]. This is specially relevant in some of the largest instances, e.g., "nine5d", where the barrier was much faster than simplex. We also see there are significant differences between solvers; for example, instance "table4" took 150.91 seconds with the simplex of CPLEX, only 6.85 with that of XPRESS, and 627 with Clp; but we got opposite results for instance "nine12". Another conclusion is that, for these LO-CTA problems, the barrier of XPRESS, which is based on the BPMPD solver [23], seems to be a very efficient option.

It was also observed that problems (14) within the lexmin optimization became more difficult for the barrier algorithm as new constraints $f_{\pi(l)}(\omega) \leq f_{\pi(l)}^{*}$ were added. This is illustrated in Table 4 , which reports for the subset of largest instances the CPU time and number of iterations (in brackets) of the interior-point and simplex algorithms of XPRESS for the four subproblems solved (using the order $\pi=(4,2,3,1)$ ). The interior-point CPU time does not include the crossover (that is, the procedure for obtaining a vertex solution from the (possibly non-vertex) primal-dual solution computed by the interiorpoint algorithm); this explains why the sum of the four times does not equal the values in Table 3. Indeed, it can be seen that instances "five20b" and "five20c" exhausted the time limit because of the crossover. (Crossover can be deactivated in the application developed, but in general it provides sparser solutions - since they are basic solutions, unlike the interior-point ones - which are preferred.) It is clearly seen that as new constraints of the type $f_{\pi(l)}(\omega) \leq f_{\pi(l)}^{*}$ are added the number of interior-point iterations (and its CPU time) increases significantly. However this fact is not observed for the simplex method. Although we did not analyze in detail this different behaviour, a possible explanation would be that the feasible region is significantly reduced as we are adding new constraints (we limit the feasible region of the new problem to be the optimal face of the previous one). The central-path of the new problem, which the path-following methods implemented in 


\begin{tabular}{|c|c|c|c|c|c|c|}
\hline \multirow[b]{2}{*}{ instance } & \multicolumn{2}{|c|}{ CPLEX } & \multicolumn{2}{|c|}{ XPRESS } & \multicolumn{2}{|c|}{ Clp } \\
\hline & barrier & simplex & barrier & simplex & barrier & simplex \\
\hline australia_ABS.csp & 3.01 & 1.05 & 2.28 & 1.52 & 2.25 & 2.24 \\
\hline bts4.csp & 3429.09 & $>3600$ & $>3600$ & $>3600$ & 1273.29 & - \\
\hline cbs.csp & 0.37 & 0.65 & 0.6 & 0.48 & 0.52 & 0.5 \\
\hline dale.csp & 1.3 & 10.13 & 1.63 & 1.84 & 1.89 & 1.8 \\
\hline destatis.csp & 2.66 & 30.43 & 3.43 & 5.35 & 13 & 9.08 \\
\hline five 20b.csp & $>3600$ & $>3600$ & $>3600$ & $>3600$ & $>3600$ & $>3600$ \\
\hline five20c.csp & $>3600$ & $>3600$ & $>3600$ & $>3600$ & $>3600$ & $>3600$ \\
\hline hier13.csp & 8.48 & 39.91 & 11.64 & 27.76 & 47.56 & - \\
\hline hier13x13x13a.csp & 25.55 & 53.61 & 7.11 & 21.48 & 276.51 & 49.23 \\
\hline hier $13 \times 13 \times 13 b . c s p$ & 21.04 & 29.37 & 11.25 & 23.08 & 271.31 & 48.61 \\
\hline hier $13 \times 13 \times 13$ c.csp & 20.8 & 67.58 & 6.58 & 22.92 & 306.3 & 48.61 \\
\hline hier13x13x13d.csp & 14.81 & 72.88 & 12.43 & 28.65 & 75.55 & - \\
\hline hier $13 \times 13 \times 13$ e.csp & 15.63 & 45.46 & 9.8 & 24.73 & 59.5 & - \\
\hline hier $13 \times 13 \times 7$ d.csp & 1.83 & 3.24 & 1.14 & 2.81 & 2.06 & 1.69 \\
\hline hier $13 \times 7 \times 7$ d.csp & 0.34 & 0.26 & 0.32 & 0.29 & 0.24 & 0.22 \\
\hline hier16.csp & 822.08 & 1150.16 & 112.79 & 287.47 & $>3600$ & - \\
\hline hier16x16x16a.csp & 114.35 & 240.82 & 33.11 & 196.05 & 374.96 & - \\
\hline hier $16 \times 16 \times 16 b . c s p$ & 112.43 & 223 & 31.92 & 120.15 & 429 & - \\
\hline hier $16 \times 16 \times 16$ c.csp & 68.24 & 285.45 & 37.91 & 127.1 & 385.2 & - \\
\hline hier $16 \times 16 \times 16$ d.csp & 95.7 & 179.76 & 32.07 & 106.36 & 464.43 & - \\
\hline hier $16 \times 16 \times 16$ e.csp & 61.83 & 291.04 & 32.31 & 99.05 & 344 & - \\
\hline nine12.csp & 784.24 & 1618.35 & $>3600$ & 2740.72 & 928.18 & 713.59 \\
\hline nine 5 d.csp & 68 & 2049.09 & 130.46 & 1363.12 & $>3600$ & 412.81 \\
\hline ninenew.csp & 60.89 & 171.7 & 62.41 & 195.86 & 159.05 & 116.14 \\
\hline osorio.csp & 1.88 & 0.57 & 1.92 & 2.84 & 1.8 & 1.75 \\
\hline sbs2008_C.csp & 0.5 & 0.88 & 0.57 & 0.26 & 2.12 & 1.17 \\
\hline sbs2008_D_b.csp & 5.41 & 12.03 & 4.31 & 1.73 & 27.34 & 25.14 \\
\hline sbs $2008^{-}$E.csp & 0.12 & 0.08 & 0.19 & 0.15 & 0.13 & 0.1 \\
\hline table1.csp & 0.24 & 0.51 & 0.34 & 0.51 & 0.58 & 0.41 \\
\hline table3.csp & 6.5 & 52.71 & 4.86 & 6.6 & 170.97 & 944.8 \\
\hline table4.csp & 3.77 & 150.91 & 4.8 & 6.85 & 167.31 & 627.86 \\
\hline table5.csp & 3.25 & 49.15 & 4.93 & 6.63 & 165.09 & 510.19 \\
\hline table6.csp & 0.19 & 0.55 & 0.36 & 0.52 & 0.39 & 0.5 \\
\hline table7.csp & 0.16 & 0.11 & 0.21 & 0.16 & 0.14 & 0.1 \\
\hline table8.csp & 0.07 & 0.07 & 0.18 & 0.18 & 0.03 & 0.04 \\
\hline targus.csp & 0.01 & 0.01 & 0.09 & 0.09 & 0.01 & 0.02 \\
\hline toy3dsarah.csp & 0.34 & 1.52 & 0.47 & 0.22 & 6.63 & 0.84 \\
\hline two5in6.csp & 145.82 & 237.9 & 47.01 & 185.44 & 210.82 & 103.86 \\
\hline
\end{tabular}

Table 3. CPU time with interior-point and simplex algorithms of solvers CPLEX, XPRESS and Clp, using order $\pi=(4,2,3,1)$, allowing a $2 \%$ of deviations in cell values, and the random heuristic to fix the protection direction of sensitive cells. In italics the fastest run between interior-point and simplex for each solver; in italic and boldface, the fastest run for all the solvers.

\begin{tabular}{|c|c|c|c|c|c|c|c|c|}
\hline \multirow[b]{2}{*}{ instance } & \multicolumn{4}{|c|}{ barrier } & \multicolumn{4}{|c|}{ simplex } \\
\hline & $f_{4}$ & $f_{2}$ & $f_{3}$ & $f_{1}$ & $f_{4}$ & $f_{2}$ & $f_{3}$ & $f_{1}$ \\
\hline bts4 & $0(0)$ & $11(23)$ & $23(51)$ & $31(85)$ & $0(0)$ & $145(255 \mathrm{~K})$ & $1167(542 \mathrm{~K})$ & - \\
\hline five $20 b$ & $0(0)$ & $316(24)$ & - & - & $0(0)$ & - & - & - \\
\hline five $20 b$ & $0(0)$ & $475(30)$ & - & - & $0(0)$ & - & - & — \\
\hline hier16 & $0(0)$ & $8(20)$ & $23(37)$ & $30(70)$ & $0(0)$ & $38(85 \mathrm{~K})$ & $145(116 \mathrm{~K})$ & $104(70 \mathrm{~K})$ \\
\hline nine $5 \mathrm{~d}$ & $0(0)$ & $19(26)$ & $17(38)$ & $57(84)$ & $0(0)$ & $107(222 \mathrm{~K})$ & $728(310 \mathrm{~K})$ & $635(147 \mathrm{~K})$ \\
\hline nine12 & $0(0)$ & $36(29)$ & $56(49)$ & $106(101)$ & $0(0)$ & $277(280 \mathrm{~K})$ & $1982(577 \mathrm{~K})$ & $481(129 \mathrm{~K})$ \\
\hline two5in6 & $0(0)$ & $13(36)$ & $10(48)$ & $21(76)$ & $0(0)$ & $36(126 \mathrm{~K})$ & $59(49 \mathrm{~K})$ & $90(53 \mathrm{~K})$ \\
\hline
\end{tabular}

Table 4. CPU time and number of iterations (within brackets, in thousands for simplex) of XPRESS with interior-point and simplex algorithms for the solution of each particular objective in the largest instances. Barrier time does not include crossover (this explains why the time limit of 3600 seconds is not reached in instances bts 4 and nine12, unlike in Table 3 .

CPLEX, XPRESS and Clp attempt to "follow", is thus confined to a much smaller region, such that large directions can not be taken by the interior-point algorithm. This would explain the unexpectedly large number of interior-point iterations of the last subproblem with $f_{1}$. On the other hand, this reduction in the size of the feasible region may not negatively affect to simplex; indeed, reducing the number of feasible vertices may even be a benefit, as shown in some instances of Table 4 . 


\begin{tabular}{|c|c|c|c|c|c|c|c|c|c|}
\hline \multirow[t]{2}{*}{ instance } & \multirow[b]{2}{*}{ perc.: } & \multicolumn{4}{|c|}{ CPU time } & \multicolumn{4}{|c|}{$f_{2}$} \\
\hline & & $2 \%$ & $5 \%$ & $10 \%$ & $100 \%$ & $2 \%$ & $5 \%$ & $10 \%$ & $100 \%$ \\
\hline australia_ABS & & 2.25 & 1.97 & 2.21 & $\dagger$ & 16.31 & 16.31 & 16.31 & $\dagger$ \\
\hline bts4 & & $>3600$ & 1628 & 1052 & 821 & 1582 & 1582 & 1582 & 1582 \\
\hline $\operatorname{cbs}$ & & 0.6 & 0.6 & 0.6 & 0.53 & 15.96 & 15.96 & 15.96 & 15.96 \\
\hline dale & & 1.63 & 1.54 & 1.5 & 1.35 & 0 & 0 & 0 & 0 \\
\hline destatis & & 3.43 & 3.16 & 3.88 & 4.19 & 528 & 528 & 528 & 528 \\
\hline five $20 \mathrm{~b}$ & & $>3600$ & $>3600$ & $>3600$ & $>3600$ & 0 & 0 & 0 & 0 \\
\hline five20c & & $>3600$ & $>3600$ & $>3600$ & $>3600$ & 0 & 0 & 0 & 0 \\
\hline hier13 & & 11.6 & 9.83 & 10.03 & 6.58 & 362 & 362 & 362 & 362 \\
\hline hier13x13x13a & & 7.12 & 6.1 & 4.45 & 5.09 & 340 & 340 & 340 & 340 \\
\hline hier13x13x13b & & 11.3 & 5.14 & 3.48 & 4.6 & 340 & 340 & 340 & 340 \\
\hline hier $13 \times 13 \times 13 c$ & & 6.58 & 6.28 & 4.06 & 4.34 & 340 & 340 & 340 & 340 \\
\hline hier13x13x13d & & 12.4 & 7.27 & 6.4 & 3.88 & 511 & 511 & 511 & 511 \\
\hline hier13x13x13e & & 9.8 & 14.44 & 6.41 & 4.79 & 562 & 562 & 562 & 562 \\
\hline hier13x13x7d & & 1.15 & 1.72 & 0.81 & 0.77 & 146 & 146 & 146 & 146 \\
\hline hier $13 \times 7 \times 7 d$ & & 0.32 & 0.28 & 0.27 & 0.27 & 60.58 & 60.58 & 60.58 & 60.58 \\
\hline hier16 & & 113 & 49.51 & 26.99 & 35.88 & 300 & 300 & 300 & 300 \\
\hline hier $16 \times 16 \times 16 a$ & & 33.08 & 28.97 & 20.54 & 20.88 & 490 & 490 & 490 & 490 \\
\hline hier16x16x16b & & 31.85 & 36.4 & 20.38 & 20.85 & 490 & 490 & 490 & 490 \\
\hline hier $16 \times 16 \times 16 c$ & & 37.94 & 29.69 & 21.08 & 22.41 & 490 & 490 & 490 & 490 \\
\hline hier16x16x16d & & 32.04 & 24.95 & 25.94 & 23.13 & 735 & 735 & 735 & 735 \\
\hline hier $16 \times 16 \times 16 e$ & & 32.16 & 20.34 & 21.64 & 20.11 & 735 & 735 & 735 & 735 \\
\hline nine12 & & $>3600$ & 187 & 207 & 178 & 684 & 684 & 684 & 684 \\
\hline nine $5 d$ & & 131 & 99.33 & 116 & 74.59 & 3650 & 3650 & 3650 & 3650 \\
\hline ninenew & & 62.31 & 76.02 & 54.6 & 68.61 & 662 & 662 & 662 & 662 \\
\hline osorio & & 1.91 & 1.92 & 1.93 & 1.38 & 0 & 0 & 0 & 0 \\
\hline sbs $2008 \_C$ & & 0.57 & 0.57 & 0.57 & $\dagger$ & 28152 & 28152 & 28152 & $\dagger$ \\
\hline sbs2008_D_b & & 4.32 & 4.06 & 4.56 & $\dagger$ & 77515 & 77515 & 77515 & $\dagger$ \\
\hline sbs2008_E ${ }^{-}$ & & 0.18 & 0.21 & 0.21 & $\dagger$ & 9985 & 9985 & 9985 & $\dagger$ \\
\hline table1 & & 0.36 & 0.28 & 0.31 & 0.35 & 289 & 289 & 289 & 289 \\
\hline table3 & & 4.86 & 3.87 & 4.06 & 2.53 & 570 & 570 & 570 & 570 \\
\hline table4 & & 4.83 & 3.49 & 5.61 & 2.4 & 570 & 570 & 570 & 570 \\
\hline table5 & & 4.92 & 3.38 & 4.2 & 2.49 & 570 & 570 & 570 & 570 \\
\hline table6 & & 0.37 & 0.34 & 0.3 & 0.59 & 279 & 279 & 279 & 279 \\
\hline table7 & & 0.21 & 0.21 & 0.19 & 0.18 & 4.575 & 4.575 & 4.575 & 4.575 \\
\hline table8 & & 0.21 & 0.19 & 0.22 & 0.15 & 0 & 0 & 0 & 0 \\
\hline targus & & 0.1 & 0.08 & 0.1 & 0.1 & 0.1148 & 0.1148 & 0.1148 & 0.1148 \\
\hline toy3dsarah & & 0.48 & 0.49 & 0.48 & 0.55 & 657301 & 657301 & 657301 & 657301 \\
\hline two5in6 & & 47.01 & 47.16 & 56.11 & 50.63 & 1591 & 1591 & 1591 & 1591 \\
\hline
\end{tabular}

Table 5. CPU time and value of $f_{2}$ for different maximum percentages of deviation in cell values, using the interior-point of XPRESS as solver, order $\pi=(4,2,3,1)$, and the random heuristic to fix the protection direction of sensitive cells. In boldface, the fastest run.

\subsection{Evaluation of allowed percentage of deviations in cell values}

Tables 5 and 6 report the CPU time of the lexmin optimization, and the optimal value of objective functions $f_{1}, f_{2}$ and $f_{3}$, allowing different percentage of deviations in cells values $(2 \%, 5 \%, 10 \%$ and $100 \%)$. Objective $f_{4}$ is not reported since it was 0 in all the executions (as discussed above). The order $\pi=(4,2,3,1)$ and the interior-point of XPRESS were used in these runs. The fastest execution is marked in boldface. A time limit of 3600 seconds was also used; when the time limit is reached tables 5 and 6 report the best objective function found so far. Notice that $f_{2}$ reached the same value, independently of the percentage, for every instance. As expected, the problems become generally easier when the percentage of allowed deviations increases, and most of the fastest executions are obtained with $10 \%$ and $100 \%$. However, and unexpectedly, the real problems by Eurostat "sbs2008_C", "sbs2008_D_b" and "sbs2008_E", and "australia_ABS" instance, were reported as infesible with $\overline{100 \%}$, while they could be easily solved with $2 \%, 5 \%$ and $10 \%$. As for the objective functions, there are not significant differences, since they report aggregated information for all the cells. However, in practice, using a tight percentage of $2 \%$ (which is the default value considered) provides protected tables where all the cells are consistently close to those of the original table. 


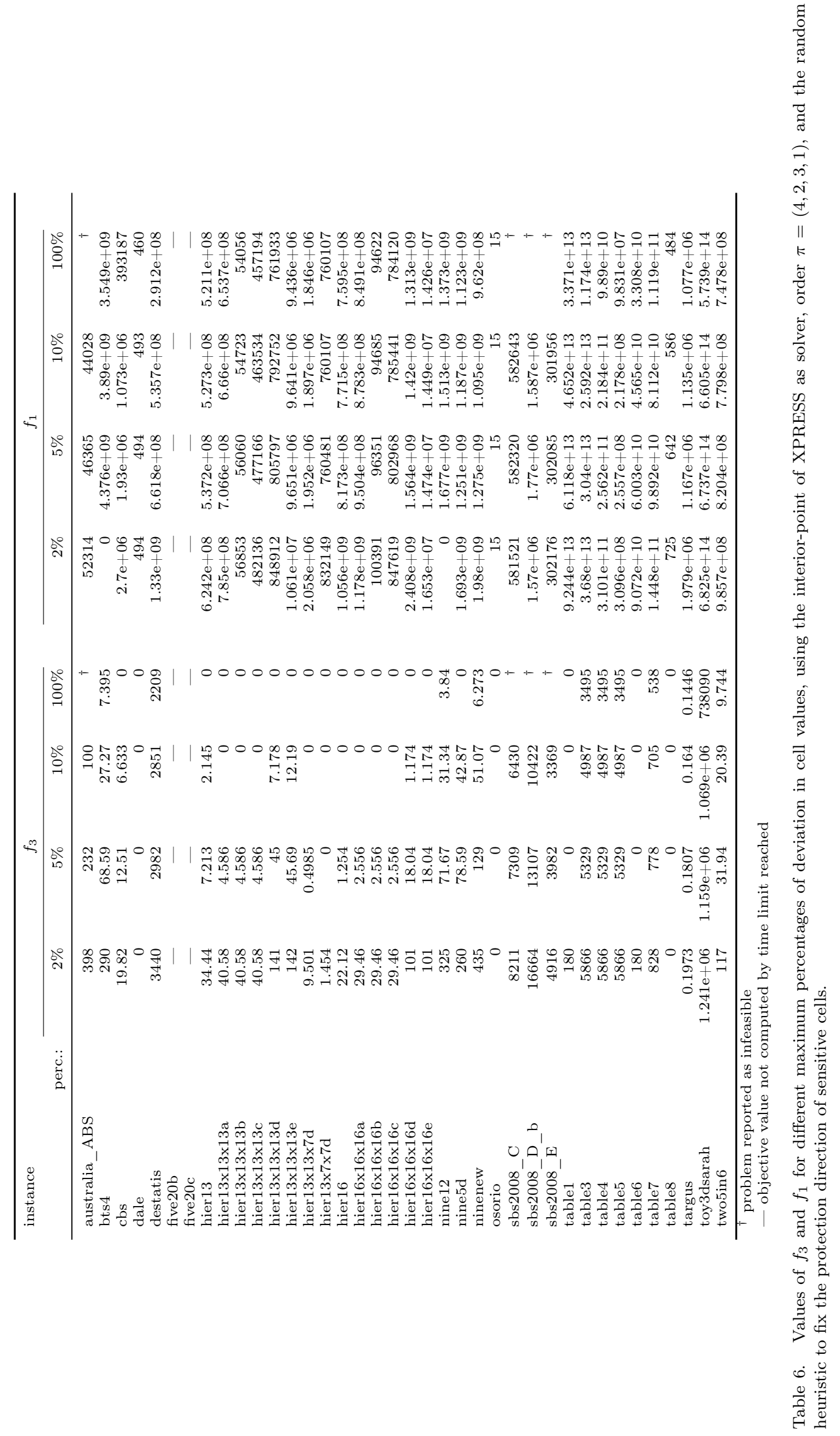




\begin{tabular}{|c|c|c|c|c|c|c|c|c|c|}
\hline \multirow[t]{2}{*}{ instance } & \multirow[b]{2}{*}{ method: } & \multicolumn{4}{|c|}{ CPU time } & \multicolumn{4}{|c|}{$f_{2}$} \\
\hline & & random & SAT & network & both & random & SAT & network & both \\
\hline australia_ABS & & 2.66 & 3.23 & * & 12.88 & 3.805 & 0 & - & $\mathbf{0}$ \\
\hline bts 4 & & $>3600$ & 3188 & 3309 & 3157 & 1582 & 42.02 & 534 & 41.47 \\
\hline $\operatorname{cbs}$ & & 0.44 & 2.73 & $*$ & 2176 & 15.96 & 0 & - & $\mathbf{0}$ \\
\hline dale & & 1.33 & 5.6 & * & * & 0 & 0 & - & - \\
\hline destatis & & 4.32 & 1.87 & 6.75 & 4.1 & 528 & 0 & 743 & $\mathbf{0}$ \\
\hline five $20 b$ & & $>3600$ & $>3600$ & $>3600$ & $>3600$ & 5742 & 26.78 & 2994 & 0 \\
\hline five20c & & $>3600$ & $>3600$ & $>3600$ & $>3600$ & 7074 & 24.73 & 3880 & 21.43 \\
\hline hier13 & & 12.17 & 8.64 & 22.23 & 10.24 & 362 & 64.73 & 85.03 & 45.25 \\
\hline hier $13 \times 13 \times 13 a$ & & 9.47 & 1.91 & 9.66 & 2.18 & 340 & 0 & 242 & o \\
\hline hier $13 \times 13 \times 13 b$ & & 9.68 & 1.85 & 9.67 & 2.03 & 340 & 0 & 242 & $\mathbf{0}$ \\
\hline hier $13 \times 13 \times 13 c$ & & 10.82 & 1.92 & 8.49 & 2.04 & 340 & 0 & 242 & 0 \\
\hline hier13x13x13d & & 11.39 & 1.92 & 11.53 & 2.04 & 511 & 0 & 362 & $\mathbf{0}$ \\
\hline hier13x13x13e & & 18.42 & 1.9 & 12.28 & 1.95 & 562 & 0 & 367 & $\mathbf{0}$ \\
\hline hier $13 \times 13 \times 7 d$ & & 1.34 & 0.38 & 1.3 & 0.36 & 146 & 0 & 174 & $\mathbf{0}$ \\
\hline hier $13 \times 7 \times 7 d$ & & 0.26 & 0.11 & 0.37 & 0.12 & 60.58 & $\mathbf{0}$ & 88.72 & $\mathbf{0}$ \\
\hline hier16 & & 58.66 & 64.82 & 102 & 32.45 & 300 & 77.43 & 206 & 39.7 \\
\hline hier $16 \times 16 \times 16 a$ & & 68.59 & 6.7 & 85.49 & 7.26 & 490 & 0 & 565 & o \\
\hline hier $16 \times 16 \times 16 b$ & & 40.84 & 6.29 & 49.96 & 6.93 & 490 & $\mathbf{0}$ & 565 & $\mathbf{0}$ \\
\hline hier $16 \times 16 \times 16 \mathrm{c}$ & & 38.92 & 6.58 & 45.1 & 7.03 & 490 & 0 & 565 & 0 \\
\hline hier $16 \times 16 \times 16 d$ & & 48.59 & 7.14 & 57.84 & 7.28 & 735 & $\mathbf{0}$ & 850 & $\mathbf{0}$ \\
\hline hier $16 \times 16 \times 16 e$ & & 47.05 & 6.6 & 60.58 & 7.14 & 735 & 0 & 850 & $\mathbf{0}$ \\
\hline nine12 & & 210 & 802 & 734 & 33.02 & 684 & 27.73 & 448 & $\mathbf{0}$ \\
\hline nine $5 d$ & & 477 & 73.61 & 703 & 17.51 & 3650 & 13.88 & 1782 & $\mathbf{0}$ \\
\hline ninenew & & 195 & 141 & 181 & 19.32 & 662 & 54.43 & 434 & $\mathbf{0}$ \\
\hline osorio & & 1.74 & 1.74 & 1.78 & 1.77 & 0 & $\mathbf{0}$ & $\mathbf{0}$ & $\mathbf{0}$ \\
\hline sbs 2008 _C & & 0.53 & 0.68 & 2.77 & 1.23 & 28156 & 1115 & 27808 & 1115 \\
\hline sbs2008_D_b & & 6.26 & 7.79 & 200 & 38.17 & 77899 & 7039 & 66104 & 7075 \\
\hline sbs2008_E $\mathrm{E}^{-}$ & & 0.13 & 0.13 & 0.3 & 0.17 & 9990 & 135 & 11108 & 135 \\
\hline table1 & & 0.34 & 0.2 & 0.43 & 0.24 & 289 & 0 & 834 & $\mathbf{0}$ \\
\hline table3 & & 5.11 & 3.23 & 11.58 & 3.05 & 570 & 9.848 & 561 & 9.848 \\
\hline table4 & & 5.05 & 2.75 & 5.74 & 3.14 & 570 & 9.848 & 561 & 9.848 \\
\hline table5 & & 4.69 & 2.96 & 7.21 & 2.72 & 570 & 9.848 & 561 & 9.848 \\
\hline table6 & & 0.27 & 0.18 & 0.38 & 0.21 & 279 & 0 & 814 & $\mathbf{0}$ \\
\hline table7 & & 0.15 & 0.08 & 0.14 & 0.08 & 4.575 & 0 & 0.6003 & $\mathbf{0}$ \\
\hline table8 & & 0.09 & 0.08 & 0.08 & 0.09 & 0 & 0 & $\mathbf{0}$ & $\mathbf{0}$ \\
\hline targus & & 0.03 & 0.03 & 0.03 & 0.03 & 0.1148 & 0 & $\mathbf{0}$ & $\mathbf{0}$ \\
\hline toy3dsarah & & 0.49 & 0.27 & 0.85 & 0.32 & 657301 & 0 & 455360 & $\mathbf{0}$ \\
\hline two5in6 & & 66.01 & 7.51 & 175 & 7.91 & 1591 & 0 & 847 & 0 \\
\hline
\end{tabular}

— objective value not computed, either by time limit reached or by heuristic failure

Table 7. CPU time and value of $f_{2}$ with the four heuristics for fixing the protection direction of sensitive cells, using the interior-point of CPLEX as solver, order $\pi=(4,2,3,1)$, and allowing a $2 \%$ of deviations in cell values. In boldface, the run with the best quality solution.

\subsection{Evaluation of procedures to fix protection directions of sensitive cells}

Tables 7 and 8 report the CPU time of the lexmin optimization and the optimal value of each objective function (excluding $f_{4}$, which is not reported since it was 0 in all the runs), for the four different heuristics described in Section 5 to fix the protection directions of sensitive cells. The CPLEX homogeneous self-dual interior-point algorithm was used in these runs, instead of the standard infeasible path-following variant of Subsections 6.1 and Subsections 6.2, this explains the differences in CPU times of Tables 243 vs. Table 7 for CPLEX barrier and the random heuristic. As it will be shown in below Subsection 6.5, although the standard barrier algorithm is usually considered the fastest option, it was outperformed in some of the difficult instances by the slower - but numerically more stable when the problem is near-infeasible-homogeneous self-dual variant. The best solution is marked in boldface, according to the lexicographic order $\pi=(4,2,3,1)$ considered in those runs. A time limit of 3600 seconds was considered, which was reached in some executions. The network heuristic failed in some runs, clearly marked in the table.

From Tables 7 and 8 we see that, according to the first objective value $f_{2}$, the best solutions were obtained with the "both" variant (SAT and network), followed by the "SAT" one. We also observe that the fastest executions, in general, were also obtained with "SAT", followed by "both", whereas the other two approaches provided, for some instances, much more difficult LO problems (e.g., for table nine5d, "random" and "network" required 477 and 703 seconds, respectively, while "SAT" and "both" only needed 73.6 and 17.5). 


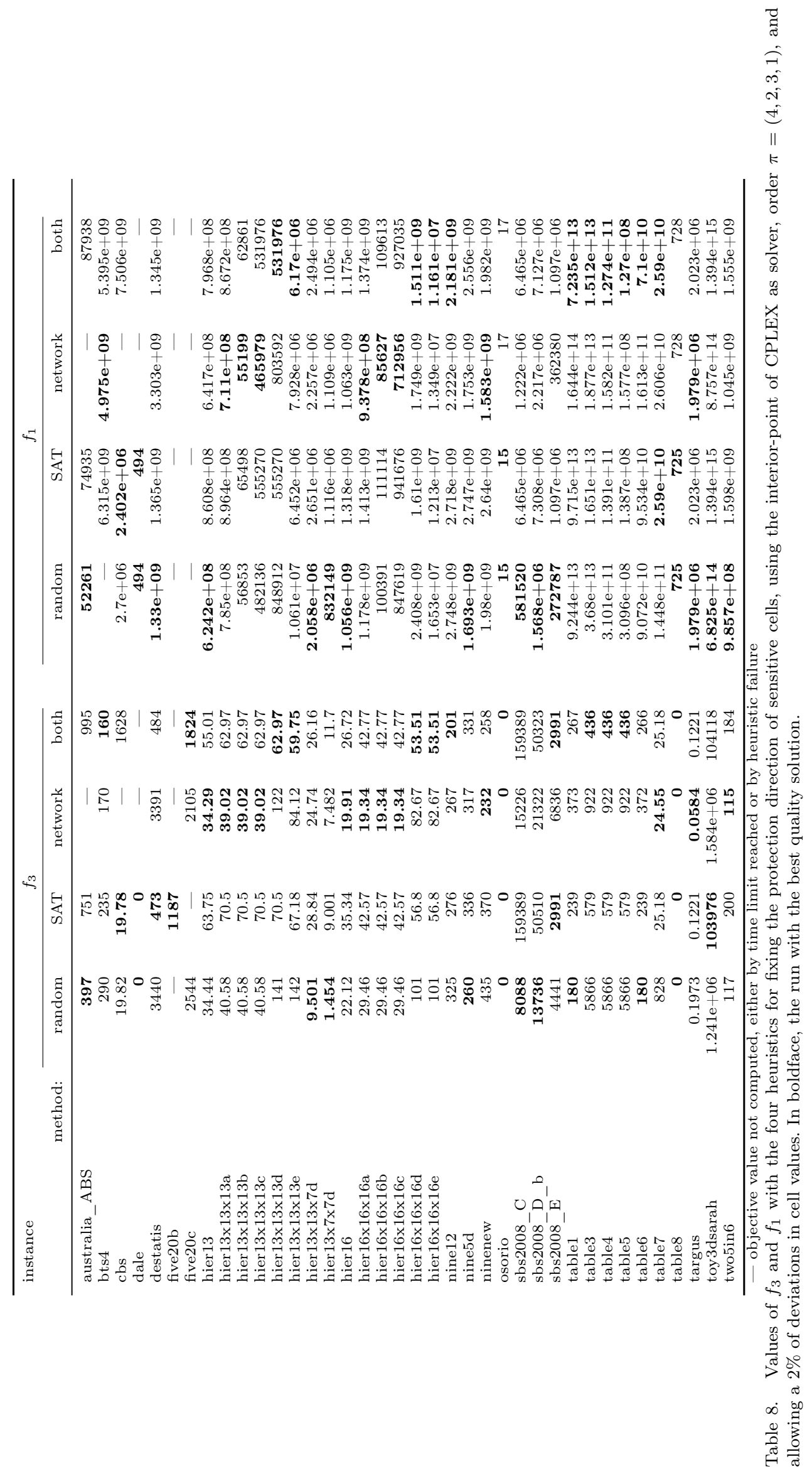




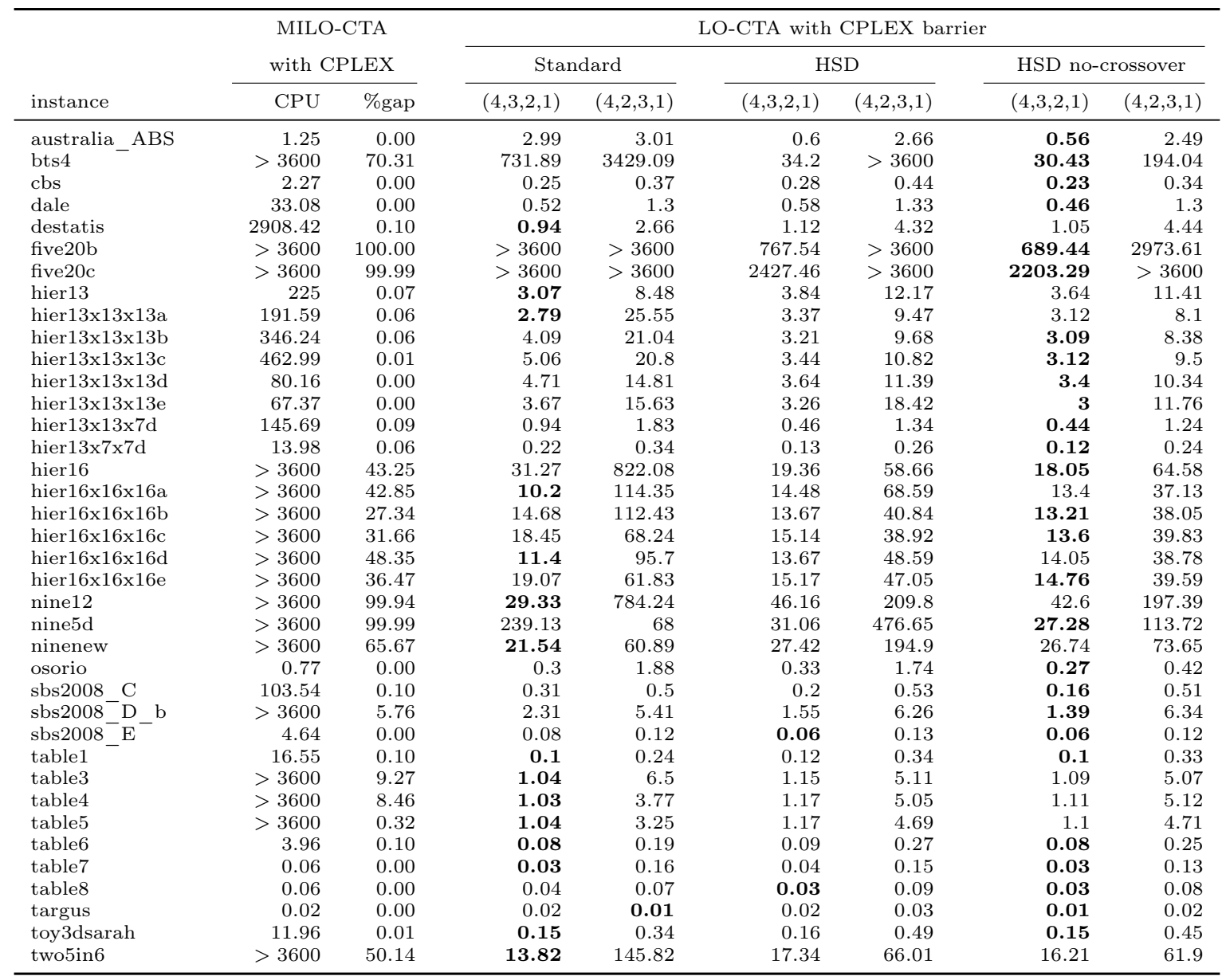

Table 9. Comparison of runtimes between MILO-CTA and LO-CTA. The CPU time and the optimality gap achieved within the 3600 seconds time limit are given for MILO-CTA. For LO-CTA, CPU times are reported for the two orders $(\pi=(4,3,2,1)$ and $\pi=(4,2,3,1))$ and three different CPLEX barrier variants (standard barrier, homogeneous self-dual barrier, and homogeneous self-dual without crossover). The fastest execution is marked in boldface.

\subsection{Comparing LO-CTA to MILO-CTA}

As it was stated in the Introduction, the purpose of LO-CTA is to provide protected tables of similar quality as those obtained with MILO-CTA, with less computational effort. In the comparison between MILO-CTA and LO-CTA we thus focus on these two criteria: efficiency and quality of the solutions.

Table 9 shows the CPU times obtained with CPLEX for MILO-CTA and LO-CTA. For LO-CTA three barrier variants were used: the standard barrier algorithm, corresponding to columns "Standard" in the table; the homogeneous self-dual interior-point (columns "HSD"); and the homogeneous self-dual without crossover (columns "HSD no-crossover"). Each barrier variant was applied to the two orders $\pi=(4,3,2,1)$ and $\pi=(4,2,3,1)$. For MILO-CTA the table also reports the optimality gap achieved; large gaps correspond to feasible sub-optimal solutions for instances that exhausted the 3600 seconds time limit. The fastest execution is marked in boldface.

Although MILO-CTA never provided the fastest run, it was very competitive in some instances (such as "australia_ABS", "cbs", "osorio", "table6", "table7" and "table8"). However, in some other cases MILO-CTA required very long executions (it even exhausted the 3600 seconds time limit) whereas LO-CTA computed a solution in one second (e.g., "destatis", "sbs2008_D_b", "table3", "table4" and "table5"). It is clearly observed that, of 


\begin{tabular}{|c|c|c|}
\hline \multirow[b]{2}{*}{ instance } & \multicolumn{2}{|c|}{ Ratio MILO-CTA/LO-CTA } \\
\hline & $(4,3,2,1)$ & $(4,2,3,1)$ \\
\hline australia ABS & 0.99 & 0.83 \\
\hline bts4 & 1.25 & 1.05 \\
\hline cbs & 0.73 & 0.69 \\
\hline dale & 0.79 & 0.79 \\
\hline destatis & 0.73 & 0.63 \\
\hline five $20 \mathrm{~b}$ & 147.6 & 103.3 \\
\hline five20c & 153.6 & 101.3 \\
\hline hier13 & 1.23 & 0.89 \\
\hline hier13x13x13a & 1.08 & 0.78 \\
\hline hier $13 \times 13 \times 13 b$ & 1.09 & 0.78 \\
\hline hier13x13x13c & 1.09 & 0.78 \\
\hline hier13x13x13d & 0.89 & 0.51 \\
\hline hier $13 \times 13 \times 13 \mathrm{e}$ & 0.99 & 0.47 \\
\hline hier $13 \times 13 \times 7 d$ & 0.95 & 0.87 \\
\hline hier $13 \times 7 \times 7 d$ & 0.85 & 0.80 \\
\hline hier16 & 0.95 & 0.80 \\
\hline hier16x16x16a & 0.96 & 0.75 \\
\hline hier16x16x16b & 0.91 & 0.73 \\
\hline hier16x16x16c & 0.91 & 0.74 \\
\hline hier16x16x16d & 0.94 & 0.63 \\
\hline hier16x16x16e & 0.83 & 0.57 \\
\hline nine12 & 151.9 & 119.6 \\
\hline nine $5 d$ & 251.7 & 210.3 \\
\hline ninenew & 1.06 & 0.80 \\
\hline osorio & 1.00 & 1.00 \\
\hline sbs2008_C & 2.90 & 2.11 \\
\hline $\operatorname{sbs} 2008^{-} \mathrm{D} \quad \mathrm{b}$ & 2.11 & 1.60 \\
\hline sbs2008_E & 1.43 & 0.89 \\
\hline table1 & 0.62 & 0.58 \\
\hline table3 & 1.07 & 0.54 \\
\hline table4 & 1.04 & 0.52 \\
\hline table5 & 1.15 & 0.58 \\
\hline table6 & 0.61 & 0.56 \\
\hline table7 & 1.29 & 0.18 \\
\hline table8 & 1.16 & 1.16 \\
\hline targus & 1.00 & 1.00 \\
\hline toy3dsarah & 1.84 & 1.32 \\
\hline two5in6 & 1.13 & 0.94 \\
\hline
\end{tabular}

Table 10. Ratio of distances between original and protected tables, where the numerator is the distance obtained with MILO-CTA and the denominator comes from LO-CTA. A value greater than one means that the table protected with LO-CTA was closer to the original table (thus better) than the table generated by MILO-CTA.

the six LO-CTA combinations tested, the most efficient was the homogeneous self-dual barrier without crossover and with order $\pi=(4,3,2,1)$ (it was the fastest option in 27 out of 38 instances). It is also worth noting that the two homogeneous self-dual columns (with and without crossover) for order $\pi=(4,3,2,1)$ were the only variants able to solve all the instances within the time limit. Indeed, and surprisingly, the homogenous selfdual algorithm outperformed the standard barrier - which is considered the most efficient interior-point algorithm - in most cases. A possible explanation can be found in the extra constraints added to the lexicographic optimization problem (14): they make the problem near-infeasible, and in those situations the homogeneous self-dual method is known to be numerically more stable. It is also seen that in some cases the crossover significantly increased the solution time: for example, for instance "bts4" and order $\pi=(4,2,3,1)$, we reduced from more than 3600 to only 194.04 seconds when the crossover was deactivated. All in all, in general, it can be concluded that LO-CTA outperformed MILO-CTA in terms of running times.

Unlike for the CPU times, there is not a clear and well defined criterion to compare the quality of solutions provided by different protection methods in the field of statistical disclosure control. We thus considered the difference in absolute value, between the original and the protected table, that is, $\sum_{i \in \mathcal{N}}\left|x_{i}-a_{i}\right|$, which is related to $f_{1}$. We proceeded as follows. Firstly, this measure was obtained for both MILO-CTA and LO-CTA with orders $\pi=(4,3,2,1)$ and $\pi=(4,2,3,1)$ (using either standard barrier, homogeneous self-dual or homogeneous self-dual without crossover, since they provided the same or very similar solutions). Secondly, the ratio of these measures between MILO-CTA and LO-CTA was 


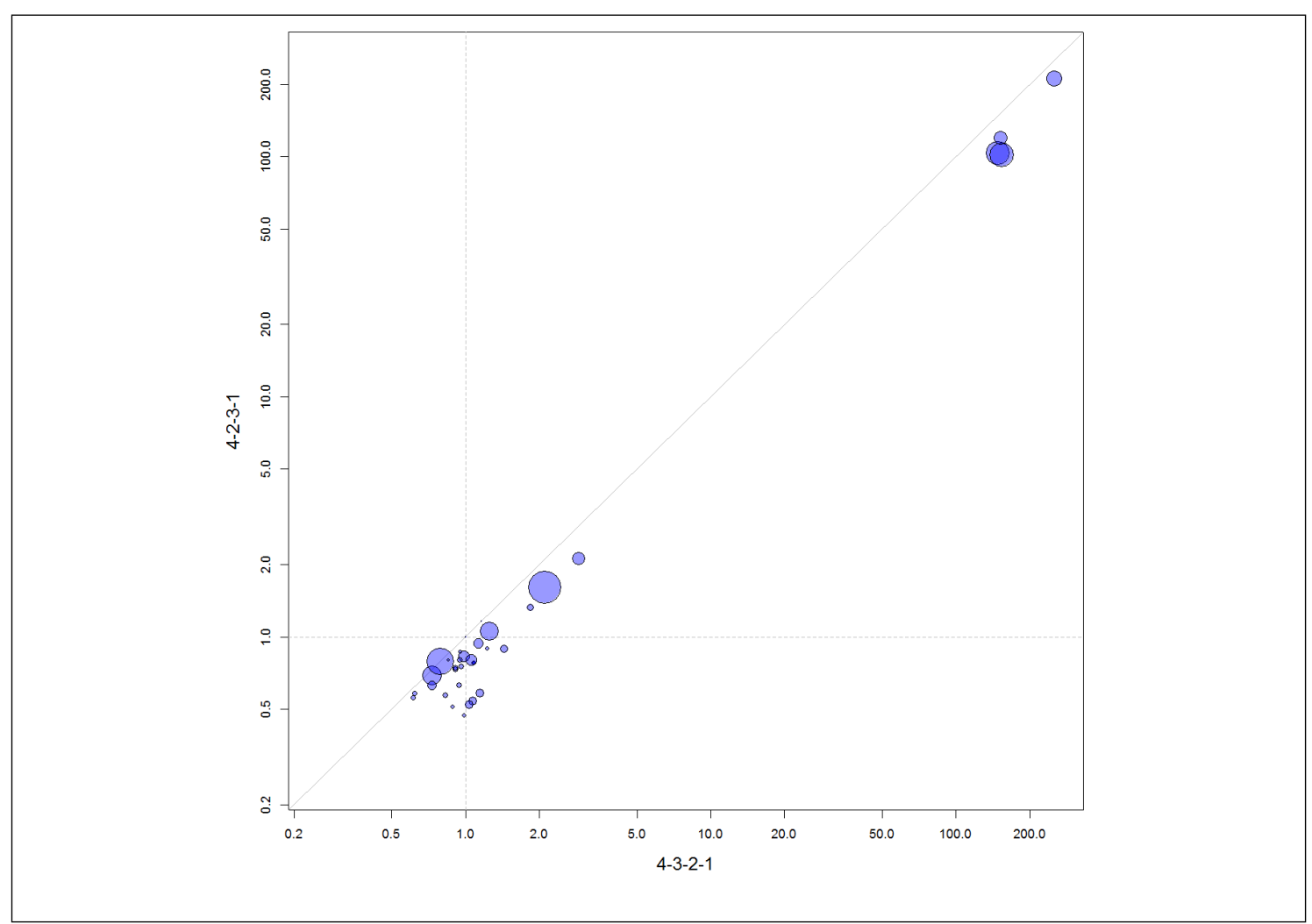

Figure 6.: Plot of ratios of $\sum_{i \in \mathcal{N}}\left|x_{i}-a_{i}\right|$ between MILO-CTA and LO-CTA with the orders $\pi=(4,3,2,1)$ (horizontal axis) and $\pi=(4,2,3,1)$ (vertical axis). Ratios greater than one mean LO-CTA behaved better than MILO-CTA. Each instance is associated with a circle, whose area is proportional to the number of sensitive cells of the instance.

computed. These ratios are shown in Table 10, Ratios greater than one mean that LOCTA provided a solution of higher quality than MILO-CTA (i.e., the protected table was closer to the original one). It is seen that LO-CTA clearly ourperformed MILO-CTA in some instances (among them some of the largest ones: "five20b", "five20c", "nine12" and "nine $5 \mathrm{~d}$ "). It could be argued that this is due to the changes in constraints and bounds done by LO-CTA. However this is not the case for two reasons: (1) First, the relative changes made by LO-CTA in constraints and bounds were very small; for example, for "five20b" - one of the largest and most difficult instances - the average relative change in constraints was 0.016 , with only four out of 52983 constraints (associated with marginal cell values) with a relative change greater than 1 (the maximum being 2.46). (2) And second, the MILO-CTA approach also reported as optimal some tables that were infeasible (some cells were unprotected, that is, their protection levels were violated); this is due to the big-M constraints of (2) and the coexistence of very small and very large cells in the same table. For example, the number of unprotected cells was 139, 60 and 49 for, respectively, "bts4", "table5" and "sbs2008_D_b" (up to 14-infeasible - tables with unprotected cells were reported as optimal by MILO-CTA).

Figure 6 shows graphically the information in Table 10 , in which each circle is associated with an instance, the coordinates of the center of the circle being the two ratios shown in the table. The areas of the circles are proportional to the number of sensitive cells of the table (in theory, the larger the number of sensitive cells, the more difficult should be the MILO-CTA problem). The line $x=y$ of Figure 6 would correspond to instances where the behaviour of LO-CTA is the same for orders $\pi=(4,3,2,1)$ and $\pi=(4,2,3,1)$. The half-plane $x>y$ is associated with instances where the order $\pi=(4,3,2,1)$ provided better solutions than $\pi=(4,2,3,1)$ : it is seen that this is the case for all the executions. 
This means that $\pi=(4,3,2,1)$ not only provides the fastest executions, but it also gives better solutions than $\pi=(4,2,3,1)$. We also see that a significant number of instances is located in the half-plane $x>1$, that is, LO-CTA with $\pi=(4,3,2,1)$ outperformed MILO-CTA, and by a large margin in the extreme instances. On the other hand, when MILO-CTA was better (instances in half-plane $x<1$ ) the $x$-coordinates of the points were always in the interval $[0.7,1]$.

\section{Conclusions}

The formulation and solution of the new multiobjective LO-CTA data protection problems opens challenges and opportunities. Compared to the original MILO-CTA formulation, it allows to compute a publishable table of similar quality by just solving up to four LO problems. In addition, for different preference orders it is possible to generate different Pareto solutions, each of them with some particular feature. On the other hand, depending on the table it may be needed to tune not only the preference order, but also the algorithm, solver, and allowed percentage of deviations. The resulting problems are challenging for today LO solvers, and they become harder as additional constraints are added during the lexicographic method. Since much larger tables can be easily generated from the current volume of data stored by NSAs, this field of application is a source of huge instances for LO solvers.

\section{Acknowledgments}

This work has been supported by MINECO/FEDER grant MTM2015-65362-R, and grant FP7-INFRA-2010-262608 of the European Union. The authors thank the two anonymous reviewers for their insightful comments, which significantly improved the manuscript.

\section{References}

[1] M. Bacharach, Matrix rounding problems, Management Science, 9, 732-742, 1966.

[2] B. De Backer, F. Didier, E. Guére, Glop: An open-source linear programming solver, communication at 22nd International Symposium on Mathematical Programming, Pittsburgh, 2015.

[3] D. Baena, J. Castro, J. A. González, Fix-and-relax approaches for controlled tabular adjustment, Computers \& Operations Research, 58, 41-52, 2015.

[4] J. Castro, Minimum-distance controlled perturbation methods for large-scale tabular data protection, European Journal of Operational Research, 171, 39-52, 2006.

[5] J. Castro, A shortest-paths heuristic for statistical data protection in positive tables, INFORMS Journal on Computing, 19(4), 520-533, 2007.

[6] J. Castro, Recent advances in optimization techniques for statistical tabular data protection, European Journal of Operational Research, 21, 257-269, 2012.

[7] J. Castro, On assessing the disclosure risk of controlled adjustment methods for statistical tabular data, International Journal of Uncertainty, Fuzziness and Knowledge-Based Systems, 20, 921-941, 2012.

[8] J. Castro, J.A. González, A tool for analyzing and fixing infeasible RCTA instances. Lecture Notes in Computer Science, 6344, 17-28, 2010.

[9] J. Castro, A. Frangioni, C. Gentile, Perspective reformulations of the CTA problem with $L_{2}$ distances, Operations Research, 62(4), 891-909, 2014.

[10] J.W. Chinneck, Feasibility and Infeasibility in Optimization: Algorithms and Computational Methods, Springer, 2008.

[11] R.A. Dandekar (2003), Energy Information Administration, Department of Energy, USA. Personal communication.

[12] R.A. Dandekar and L.H. Cox (2002), Synthetic tabular data: An alternative to complementary cell suppression, manuscript, Energy Information Administration, US Department of. Energy. 
[13] P.-P. de Wolf, A. Hundepool, S. Giessing, J.J. Salazar, J. Castro, $\tau$-Argus User's Manual, Statistics Netherlands, 2014. Available online at http://neon.vb.cbs.nl/casc/Software/TauManualV4.1. pdf.

[14] J. Forrest, J. Hall, Clp COIN-OR solver, available online at https://projects.coin-or.org/Clp.

[15] A. Geoffrion, Proper efficiency and the theory of vector maximization. Journal of Mathematical Analysis and Applications, 22, 618-630, 1968.

[16] S. Giessing, Pre-tabular perturbation with controlled tabular adjustment: some considerations, Lecture Notes in Computer Science, 8744, 48-61, 2014.

[17] J.A. González, J. Castro, A heuristic block coordinate descent approach for controlled tabular adjustment, Computers \& Operations Research, 38, 1826-1835, 2011.

[18] M.S. Hernández, J.J. Salazar, Enhanced controlled tabular adjustment, Computers \& Operations Research, 43, 61-67, 2014.

[19] A. Hundepool, The Argus software in CENEX, Lecture Notes in Computer Science, 4302, 334-346, 2006.

[20] A. Hundepool, J. Domingo-Ferrer, L. Franconi, S. Giessing, R. Lenz, J. Naylor, E. Schulte Nordholt, G. Seri, P.-P. de Wolf, Handook on Statistical Disclosure Control v1.2, Network of Excellence in the European Statistical System in the field of Statistical Disclosure Control, 2010. Available online at http://neon.vb.cbs.nl/casc/handbook.htm

[21] A. Hundepool, J. Domingo-Ferrer, L. Franconi, S. Giessing, E. Schulte Nordholt, K. Spicer, P.-P. de Wolf, Statistical Disclosure Control. Chichester, Wiley, 2012.

[22] H. Kuhn, A. Tucker, Nonlinear programming. In J. Neyman, editor, Proceedings of the Second Berkeley Symposium on Mathematical Statistics and Probability, pages 481-492, 1951. University of California Press, Berkeley, California.

[23] Cs. Mészáros, The BPMPD interior point solver for convex quadratic programming problems, Optimization Methods and Software, 11, 431-449, 1999.

[24] H. Mittelmann, Decision tree for optimization software, available online at http://plato.asu.edu/ guide.html

[25] C.M. O'Keefe, P. Gould, T. Churches, Comparison of two remote access systems recently developed and implemented in Australia. Lecture Notes in Computer Science, 8744, 299-311, 2014.

[26] J.J. Salazar-González, Mathematical models for applying cell suppression methodology in statistical data protection, European Journal of Operational Research, 154, 740-754, 2004.

[27] K. Soininvaara, T. Oinonen, A. Nissinen, Balancing confidentiality and usability: protecting sensitive data in the case of inward Foreign AffiliaTes Statistics (FATS), Lecture Notes in Computer Science, 8744, 338-349 2014. 